\title{
Deep profiling of apoptotic pathways with mass cytometry identifies a synergistic drug combination for killing myeloma cells
}

\author{
Charis E. Teh ${ }^{1,2} \cdot$ Jia-Nan Gong ${ }^{1,2} \cdot$ David Segal $^{1,2} \cdot$ Tania Tan $^{1} \cdot$ Cassandra J. Vandenberg ${ }^{1,2} \cdot$ Pasquale L. Fedele ${ }^{1,2,3}$. \\ Michael S. Y. Low ${ }^{1,2,3} \cdot$ George Grigoriadis, ${ }^{3,4,5} \cdot$ Simon J. Harrison ${ }^{6,7} \cdot$ Andreas Strasser ${ }^{1,2} \cdot$ Andrew W. Roberts $\mathbb{D}^{1,2,6,7}$. \\ David C. S. Huang $\mathbb{1}^{1,2} \cdot$ Garry P. Nolan ${ }^{8,9} \cdot$ Daniel H. D. Gray $\mathbb{1}^{1,2} \cdot$ Melissa E. Ko ${ }^{8,9}$
}

Received: 29 March 2019 / Revised: 13 January 2020 / Accepted: 14 January 2020 / Published online: 27 January 2020

(c) The Author(s), under exclusive licence to ADMC Associazione Differenziamento e Morte Cellulare 2020

\begin{abstract}
Multiple myeloma is an incurable and fatal cancer of immunoglobulin-secreting plasma cells. Most conventional therapies aim to induce apoptosis in myeloma cells but resistance to these drugs often arises and drives relapse. In this study, we sought to identify the best adjunct targets to kill myeloma cells resistant to conventional therapies using deep profiling by mass cytometry (CyTOF). We validated probes to simultaneously detect 26 regulators of cell death, mitosis, cell signaling, and cancer-related pathways at the single-cell level following treatment of myeloma cells with dexamethasone or bortezomib. Time-resolved visualization algorithms and machine learning random forest models (RFMs) delineated putative cell death trajectories and a hierarchy of parameters that specified myeloma cell survival versus apoptosis following treatment. Among these parameters, increased amounts of phosphorylated cAMP response element-binding protein (CREB) and the pro-survival protein, MCL-1, were defining features of cells surviving drug treatment. Importantly, the RFM prediction that the combination of an MCL-1 inhibitor with dexamethasone would elicit potent, synergistic killing of myeloma cells was validated in other cell lines, in vivo preclinical models and primary myeloma samples from patients. Furthermore, CyTOF analysis of patient bone marrow cells clearly identified myeloma cells and their key cell survival features. This study demonstrates the utility of CyTOF profiling at the single-cell level to identify clinically relevant drug combinations and tracking of patient responses for future clinical trials.
\end{abstract}

These authors contributed equally: Garry P. Nolan, Daniel H. D. Gray, Melissa E. Ko

Edited by G. Melino

Supplementary information The online version of this article (https:// doi.org/10.1038/s41418-020-0498-z) contains supplementary material, which is available to authorized users.

Garry P. Nolan

gnolan@stanford.edu

$\triangle$ Daniel H. D. Gray

dgray@wehi.edu.au

1 The Walter and Eliza Hall Institute of Medical Research, Parkville, VIC, Australia

2 Department of Medical Biology, The University of Melbourne, Parkville, VIC, Australia

3 Monash Haematology, Monash Health, Clayton, VIC, Australia

4 School of Clinical Sciences at Monash Health, Monash University, Clayton, VIC, Australia

\section{Introduction}

Evasion of apoptosis is a key feature of cancer cells [1]. The intrinsic (also called the BCL-2-regulated or mitochondrial) pathway of apoptosis is regulated by interactions amongst three main factions of the BCL-2 family of proteins: the apoptosis-initiating $\mathrm{BH} 3$-only proteins

5 Centre for Cancer Research, Hudson Institute of Medical Research, Clayton, VIC, Australia

6 Clinical Haematology, Peter MacCallum Cancer Centre and Royal Melbourne Hospital, Parkville, VIC, Australia

7 Sir Peter MacCallum Department of Oncology, Melbourne University, Parkville, VIC, Australia

8 Baxter Laboratory for Stem Cell Biology, Stanford School of Medicine, Stanford, CA, USA

9 Cancer Biology Program, Stanford School of Medicine, Stanford, CA, USA 
(e.g., BIM and PUMA), the pro-survival proteins (e.g., BCL-2, MCL-1 and BCL-XL) and the apoptosis effectors, BAK and BAX [2]. Cellular stressors, such as cytotoxic drugs, cause the pro-apoptotic $\mathrm{BH} 3$-only proteins to overcome inhibition by the pro-survival proteins, leading to the activation of BAX and/or BAK which permeabilize the mitochondrial outer membrane [3,4]. This event is considered "the point of no return" for apoptosis as it induces the activation of caspases that demolish the cell $[3,5,6]$. The intrinsic apoptotic pathway can also be directly activated by $\mathrm{BH} 3$-mimetic drugs [7, 8], such as ABT-199/venetoclax, which selectively inhibits the prosurvival protein BCL-2 and is an effective new treatment for chronic lymphocytic leukemia [9]. Recent experience with venetoclax suggests that the best outcomes occur when BH3 mimetics are combined with additional anticancer agents [7-9]. The challenge now is to determine which BH3-mimetic drugs can kill which cancers and identify the most effective combinations with standard or emerging targeted therapies.

Multiple myeloma (MM) is an incurable cancer of plasma cells [10]. Corticosteroids (e.g., dexamethasone), immunomodulatory drugs (e.g., lenalidomide), and proteasome inhibitors (e.g., bortezomib) are cornerstones of MM therapy [11]. Despite their widespread use, many questions remain about the precise mechanisms of action of these drugs [10]. Moreover, how resistance to these therapies arises and how best to overcome this problem remains unclear [12]. Many studies have found that the intrinsic apoptotic pathway plays a critical role in the efficacy of MM treatments. The proteasome inhibitor, bortezomib, has been shown to induce apoptosis in myeloma cells by causing a reduction in the amount of the pro-survival protein, MCL-1, and the upregulation of proapoptotic BIM [13, 14]. Furthermore, myeloma cells readily undergo apoptosis upon genetic deletion of the pro-survival proteins MCL-1 and/or BCL-2 [15] or use of the BH3 mimetics ABT-737 (inhibiting BCL-2, BCL-XL, and BCL-W) [16, 17], ABT-199 (inhibiting BCL-2) [18], or S63845 (inhibiting MCL-1) [7]. Therefore, detailed understanding of the expression dynamics of the various BCL-2 family members in myeloma upon treatment could guide the design of optimal drug regimens that more effectively kill myeloma cells with $\mathrm{BH} 3$ mimetics.

Here we used mass cytometry, or CyTOF [19-23], to profile the dynamics of the apoptosis pathway in the MM.1S cell line treated with two standard-of-care drugs, dexamethasone or bortezomib. We resolve the major BCL-2 family proteins, key signaling pathways and cancer-relevant cellular states at the single-cell level to provide a detailed picture of apoptosis induced by these drugs, revealing distinct cellular trajectories to death. Machine learning approaches identified MCL-1 as a key feature of cells surviving dexamethasone or bortezomib treatment. Consistent with this finding, potent synergistic killing of primary patient myeloma cells was observed after treatment with dexamethasone and an inhibitor of MCL-1. Our results demonstrate the utility of mass cytometry single-cell profiling of apoptosis in identifying promising combination therapy for MM.

\section{Materials and methods}

\section{Antibody conjugates and validation}

Details on the antibody conjugates used in this study and the generation of CRISPR cell lines, western blot and flow cytometry analysis for validation are described in the Supplementary Methods and Supplementary Table 4.

\section{Cell lines}

All cell lines used were obtained from ATCC and are described in Supplementary Table 2. MM.1S cell line CyTOF experiments were performed in triplicate using the conditions outlined in Supplementary Table 3. Controls were DMSO $(0.1 \%)$ treated cells.

\section{Mass cytometry}

Cells were stained for viability with cisplatin [22] and then fixed with $1.6 \%$ paraformaldehyde (PFA: Electron Microscopy Sciences, PA, USA) for $10 \mathrm{~min}$ at room temperature. Cells were pelleted and washed once with cell staining medium (CSM, PBS with $0.5 \%$ BSA and $0.02 \%$ sodium azide). Cells were stored in CSM at $-80^{\circ} \mathrm{C}$. Thawed cells were barcoded using 20-plex palladium barcoding as described [24]. Following barcoding, cells were pelleted and washed three times with CSM, and stained with antibodies against surface markers (Supplementary Table 4 ) for $1 \mathrm{~h}$ at room temperature. Cells were permeabilized at $4{ }^{\circ} \mathrm{C}$ with methanol for $10 \mathrm{~min}$ and subsequently stained with antibodies against intracellular markers (Supplementary Table 4). Cells were stained with $125 \mathrm{nM}{ }^{191} \mathrm{Ir} /{ }^{193} \mathrm{Ir}$ DNA intercalator (Fluidigm, CA, USA) in PBS with $1.6 \%$ PFA at $4{ }^{\circ} \mathrm{C}$ overnight. Cells were washed once with CSM, three times with double-distilled water, filtered, and resuspended with EQ normalization beads immediately before analysis using a CyTOF2 or Helios CyTOF (Fluidigm, CA, USA). All FCS files generated by CyTOF were concatenated, normalized [25], and debarcoded [24] using algorithms in Supplementary Table 5. Single cells were gated using the Cytobank software [26] based on event length and ${ }^{191}$ Ir $/{ }^{193}$ Ir DNA 
content to exclude debris and doublets. Following singlecell gating, live (i.e., cisplatin ${ }^{\text {low }}$ ) cells were used for subsequent analysis from Fig. 2c onwards.

\section{Generation of heatmaps, FLOW-MAPs, and random forest model (RFM)}

Mean expression values for all proteins were calculated in Cytobank and exported into R. These values were asinh transformed and plotted in a heatmap in R. CyTOF data were visualized using the FLOWMAPR package [23] with all code to generate heatmaps and FLOW-MAPs available at https://github.com/mesako/BCL2-Publication. Random forest modeling was performed using the randomForest package in R. Details are provided in Supplementary Methods and code has been made available: https://github. com/mesako/BCL2-Publication.

\section{Tests for drug synergy}

MM.1S, OPM2, AMO1, U266, KMS-12-BM, or H929 cells were seeded in 96-well plates at $1 \times 10^{5}$ cells/well and treated with five-point 1:8 serial dilutions $(0.002-10 \mu \mathrm{M})$ of dexamethasone and either S63845, ABT-199, or A-1331852. Cell viability was determined by PI/Annexin V staining after $24 \mathrm{~h}$. The predicted additive effect was calculated using the BLISS model of fractional independence [27].

\section{Patient recruitment, cells, and analysis}

Bone marrow samples were obtained from MM patients from the Royal Melbourne Hospital and Monash Health (both in Melbourne, Australia) (Supplementary Table 6). All patients provided written informed consent and the study was approved by Human Research Ethics Committees/Institutional Review Boards: RMH (2005.008, 2012.244, 2016.305), Monash Health (HREC, project number 14406A) and the Walter and Eliza Hall Institute (G15/05).

Fresh bone marrow mononuclear cells were isolated by Ficoll density gradient centrifugation. Cells $(500,000)$ were isolated and stained with anti-CD45 V450 (BD clone HI30), anti-CD38 PE-Cy7 (BD clone HIT2), and antiCD138-APC (BD clone MI15) antibodies, with counting beads added to determine a myeloma cell count (CD45 ${ }^{-} \mathrm{CD} 38^{+} \mathrm{CD} 138^{+/-}$). Total bone marrow mononuclear cells containing at least $1000 \mathrm{CD} 45^{-} \mathrm{CD} 38^{+}$myeloma cells were then treated with $100 \mathrm{nM} \mathrm{S63845}$ or/and 100 $\mathrm{nM}$ dexamethasone. Cell viability was determined by PI/ Annexin V staining after $24 \mathrm{~h}$. Representative staining is shown in Supplementary Fig. 4 and sample calculation in Supplementary Table 7.

\section{Results}

\section{CyTOF resolution of $\mathrm{BCL}-2$ family members}

To develop probes enabling single-cell quantification of major BCL-2 family members, we identified antibodies against BCL-2, MCL-1, BCL-XL, BIM, BAX, and BAK that were compatible with PFA/methanol-based fixation and permeabilization conditions in myeloma cell lines. Antibody specificity was initially assessed by comparing flow cytometry signals in parental cell lines with negative controls rendered genetically deficient by CRISPR/Cas9-targeting (Figs. 1a, b and S1a, b). BAK and BAX exist in an inert conformation until an apoptotic stimulus activates them to permeabilize the mitochondrial outer membrane [28-30]. To identify antibodies that distinguish these states, we compared the detection of BAK and BAX in cells before and after activation with truncated (t)BID [31]. The antiBAK antibody G317-2 specifically resolved activated forms of BAK (aBAK), while anti-BAX antibody 3 detected both inert and activated forms of the BAX (Fig. 1b).

The mean intensities detected by CyTOF metal conjugates were compared with the protein expression levels detected in western blots in 12 myeloma cell lines (Fig. 1c, d). Overall, good correlation between the two detection methods was observed for MCL-1, BCL-2, BCL-XL, and $\mathrm{BIM}$, but poor correlation for BAK and especially BAX (Fig. 1e). These discrepancies likely reflect the different antibody clones used, with the western blot analysis detecting both inactive and active forms of these proteins, while the conjugates used for CyTOF were optimized for the active forms of BAX/BAK. Overall, these data demonstrate specific and robust detection of key BCL-2 family member proteins at the single-cell level by CyTOF.

\section{Dynamic changes in myeloma cell state following drug treatment}

We then sought to profile apoptotic, cell cycle, and signaling states in myeloma cells following treatment with the standard-of-care cytotoxic drugs, bortezomib or dexamethasone. MM.1S cells were chosen because they express high levels of all major pro-survival BCL-2 family proteins. Cells were treated with drugs or vehicle, then harvested in triplicate at early $(6 \mathrm{~h}$ for bortezomib and $24 \mathrm{~h}$ for dexamethasone) or late ( $24 \mathrm{~h}$ for bortezomib and $72 \mathrm{~h}$ for dexamethasone) timepoints to capture the major transitions during cell death [32, 33]. Fixed and permeabilized cells were analyzed by CyTOF for expression of BCL-2 family members, activated apoptotic mediators (active caspase- 3 and cleaved PARP), cell cycle proteins, the apoptosis-related signaling pathways NF- $\kappa B$, ERK/p38, mTOR, JAK/STAT, transcription factors (pCREB), or 
A
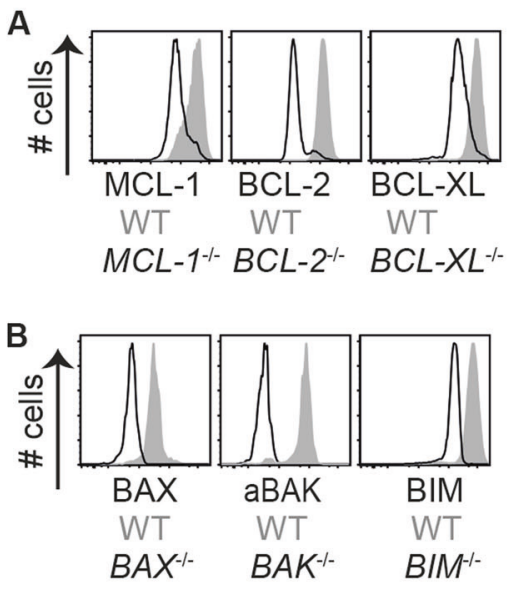

C

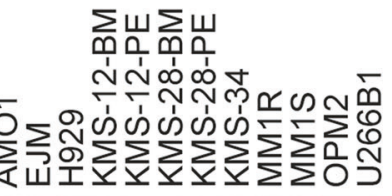

MCL-1 - - - - - - - - - $37 \mathrm{kD}$

$\mathrm{BCL}-2$ - - - - - - - $-25 \mathrm{kD}$

$\mathrm{BCL}-\mathrm{XL}----\bullet-\infty--25 \mathrm{kD}$

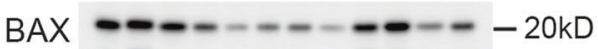

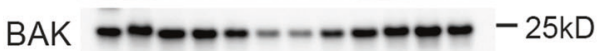

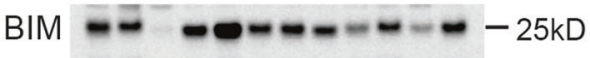

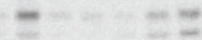

HSP70 - - - - - - - - $-75 \mathrm{kD}$

D

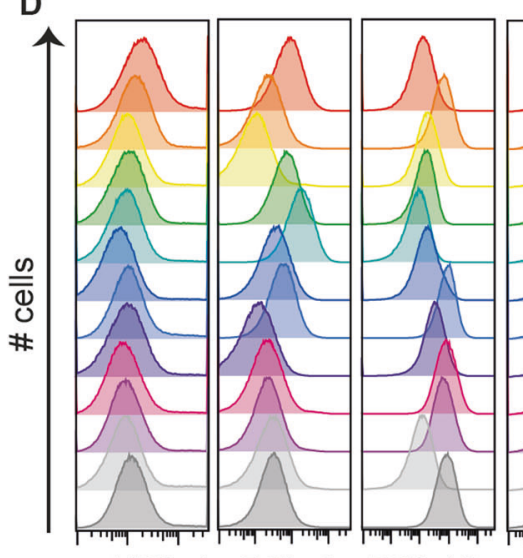

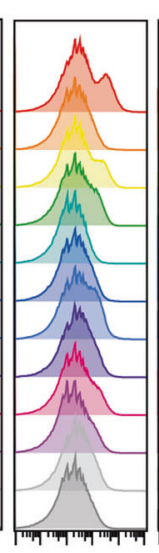

BAX

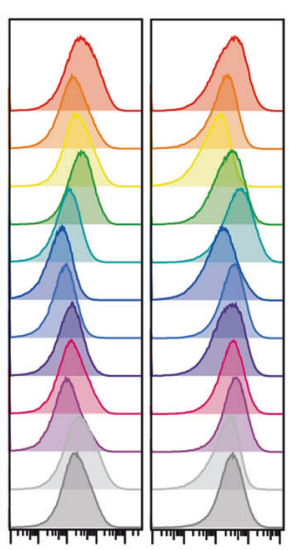

aBAK BIM

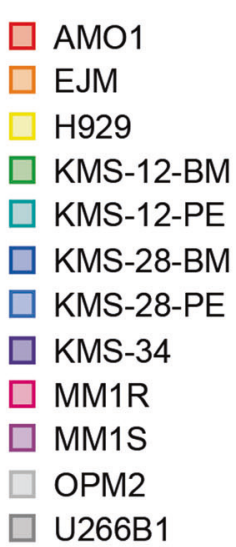

U266B1

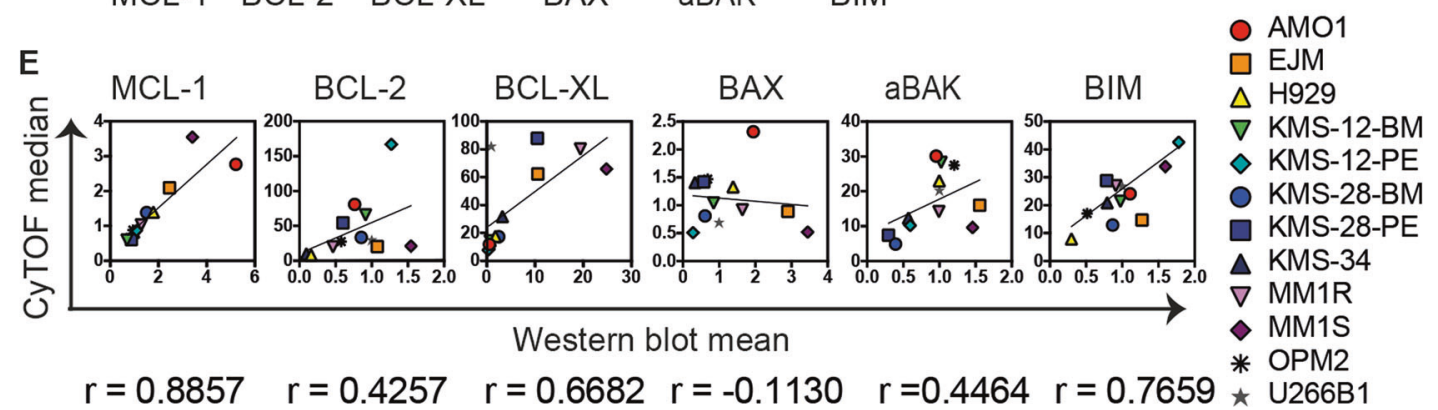

Fig. 1 CyTOF probes for detection of BCL-2 family members. a Representative histograms of the expression of the pro-survival proteins MCL-1, BCL-2, and BCL-XL in U266B1 cells (shaded gray histograms) versus isogenic CRISPR/Cas9-knockout control cells lacking these prosurvival proteins (black lines) analyzed by intracellular flow cytometry. b Representative histograms of the expression of pro-apoptotic BIM and BAX in untreated KMS-12-PE cells (shaded gray histograms) or BAK in KMS-12-PE cells treated with $100 \mathrm{nM}$ tBid (aBAK) (shaded gray histograms) versus isogenic CRISPR/Cas9-knockout controls (black lines) analyzed by intracellular flow cytometry. c Immunoblotting of cell lysates from 12 different myeloma cell lines for the indicated BCL-2 family proteins and the loading control HSP70. d Histograms of the expression of pro-survival and pro-apoptotic proteins in the same myeloma cell lines assayed by CyTOF. e Dot-plot comparisons of western blot values quantified using densitometry and median expression value by CyTOF for each BCL-2 family protein. Pearson correlation values $(r)$ are calculated for each marker using all 12 multiple myeloma cell lines. Data from $\mathbf{c}, \mathbf{d}$, and $\mathbf{e}$ are representative of two independent experiments. cancer-related proteins (p53 and c-MYC) at a single-cell level. Cisplatin was used to distinguish live (cisplatin ${ }^{\text {neg }}$ ) from dead (cisplatin ${ }^{\mathrm{pos}}$ ) cells and, as expected, both treatments induced substantial cell death over time. Accordingly, the drugs also induced high levels of early apoptotic parameters $\left(\mathrm{aCASP} 3^{\mathrm{pos}} \mathrm{cPARP}^{\mathrm{pos}}\right.$ cells) in cisplatin ${ }^{\text {neg }}$ cells (Fig. 2a).

A heatmap of the levels of various parameters in all cells showed concordance in key markers of apoptotic states and other dynamic changes over the time course (Fig. 2b). 
A
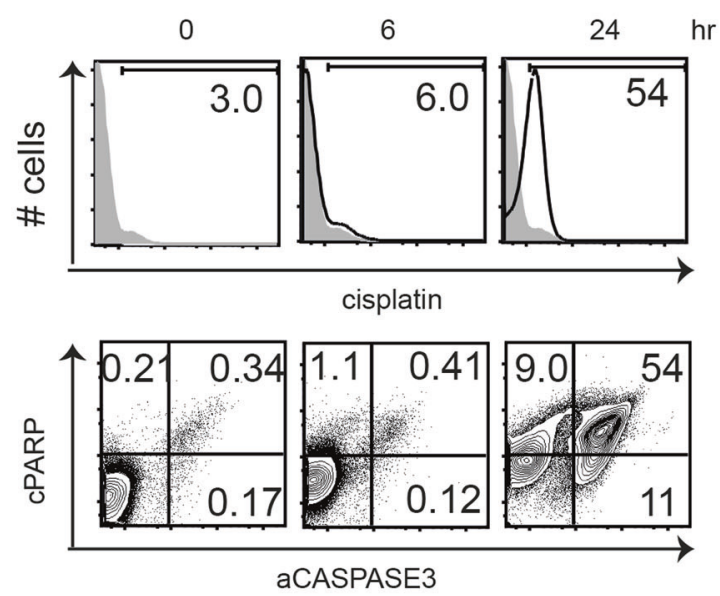

B
Dexamethasone
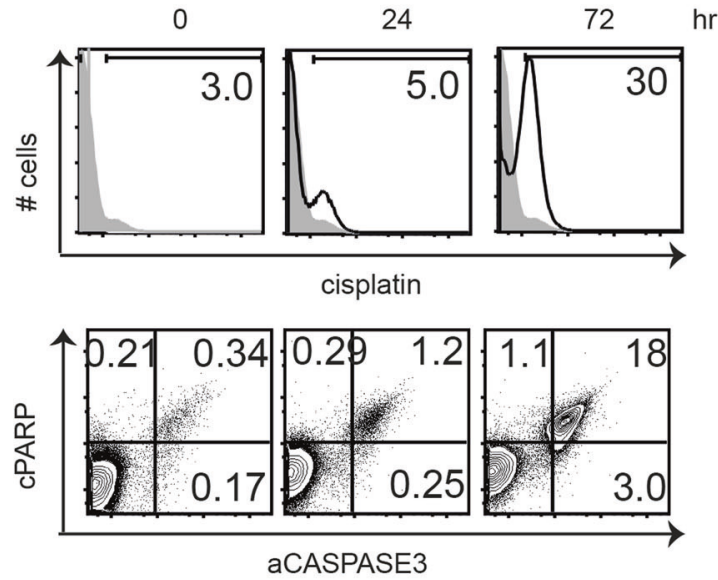

Untreated

Bortezomib $6 \mathrm{hr}$

Bortezomib $24 \mathrm{hr}$

Dexamethasone $24 \mathrm{hr}$

Dexamethasone $72 \mathrm{hr}$

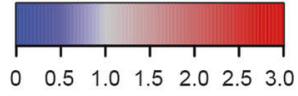

\section{C}
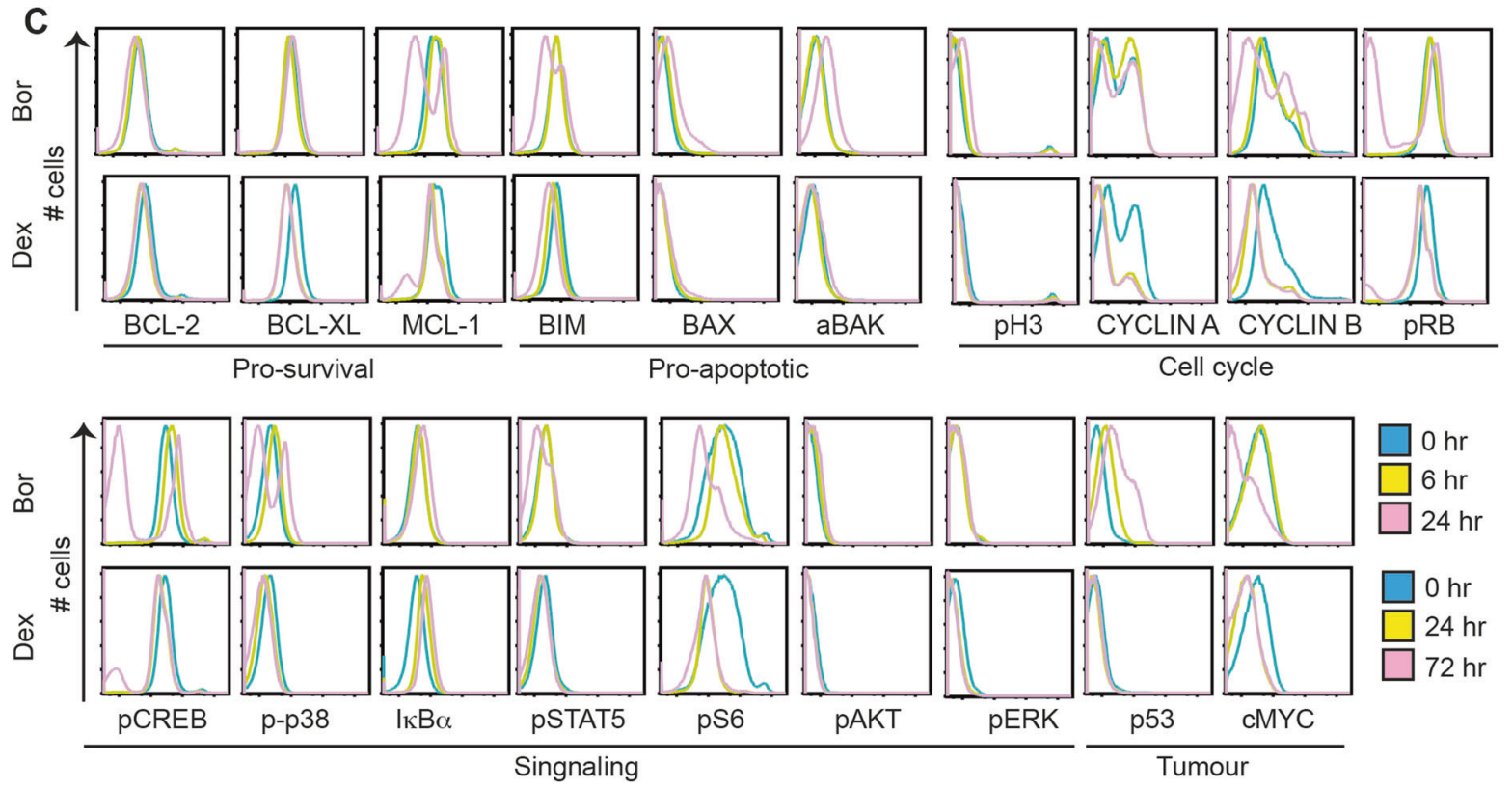

ps6

PAKT
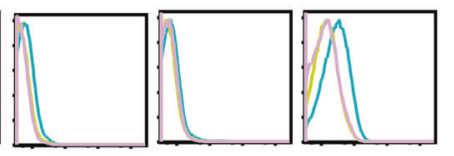

$24 \mathrm{hr}$

Histograms of the various proteins gated on cisplatin ${ }^{\text {neg }}$ cells following bortezomib or dexamethasone treatment also showed clear changes in multiple pathways (Fig. 2c), suggesting that many of these changes preceded apoptosis and were involved in the life or death "decision". The prosurvival proteins BCL-2, BCL-XL, and MCL-1 and the 
Fig. 2 Substantial changes in cell cycle and signaling states accompany apoptotic cell death following treatment of multiple myeloma cells with cytotoxic drugs. a Representative histograms of cisplatin levels (upper panels) and dot plots of cPARP versus aCASP3 levels (lower panels) in MM.1S cells over the time course of bortezomib or dexamethasone treatment. b Heatmap summary of relative protein expression levels measured by mass cytometry in MM.1S cells over time with the indicated treatments. The color scale indicates $z$-score values after $z$-score normalization across rows of the asinh ratio of change in expression levels for each marker relative to untreated cells. c Representative histograms of protein expression or phosphorylation states in MM.1S cells analyzed by mass cytometry before and after treatment with dexamethasone or bortezomib. The levels of the indicated parameters in MM.1S cells are shown following drug treatment at early (yellow histograms) or late (pink histograms) timepoints following drug treatment, or in untreated cells (blue histograms). Data are representative of three independent experiments.

pro-apoptotic protein, BIM were all decreased to varying extents (Fig. 2b, c). In addition, both drugs decreased cell cycle proteins, activation of the CREB pathway (phosphoCREB), RAS-RAF-MEK-ERK pathway (phospho-p38, phospho-ERK), PI3K/AKT pathway (phospho-S6, phospho-AKT), NF- $\mathrm{KB}$ transcription factor pathway (increased $\mathrm{I} \kappa \mathrm{B} \alpha$ ), and c-MYC (Fig. 2b, c). A distinguishing feature between the two treatments was the induction of p53 upon treatment with bortezomib but not dexamethasone (Fig. 2b, c). These data reveal dynamic changes in myeloma cells after drug treatment at the population level (e.g., phosphoS6 and MYC), but also changes restricted to subpopulations of cells (e.g., MCL-1, phospho-CREB, phospho-p38, and cell cycle regulators) (Fig. 2c). These observations prompt the question-can single-cell analysis of these features resolve the decisive step in the apoptotic response?

\section{Visualizing drug-induced temporal changes in cell state at the single-cell level}

To visualize the high-dimensional changes in the proteins measured at a single-cell level over time, we used FLOWMAP [23]. Each FLOW-MAP in Fig. 3 is representative of 10,000 individual cisplatin ${ }^{\text {neg }}$ cells clustered to form 2000 nodes in a single graph. Within each timepoint, cells are organized such that nodes with similar protein expression profiles are near while dissimilar nodes lie further apart in two-dimensional space. Each timepoint is clustered, then data from adjacent timepoints are connected to build the graph. The final FLOW-MAP displays putative trajectories of cellular responses over time with untreated, early and late timepoints denoted by blue, yellow, and pink, respectively (Fig. 3a, d). Differences in the expression of individual markers can be overlaid on these graphs (e.g., Fig. 3b, c, e, $\mathrm{f}$ ), with high expression indicated by red and low expression by blue.

Following drug treatment, cells clustered into three groups assigned as apoptotic, viable, or viable dividing based on expression of key markers (Fig. 3). The apoptotic group was aCASP $3^{\text {high }}$ and $\mathrm{cPARP}^{\text {high }}$, and this group increased at later timepoints (Fig. 3a, b, d, e). Viable cells, identified as aCASP $3^{\text {neg }}$ CPARP $^{\text {low }}$, segregated into two groups, with dividing cells distinguished as phospho- $\mathrm{H} 3^{\text {high }}$, a characteristic of cells in $\mathrm{M}$ phase (Fig. 3c, f). Other stages of cell cycle were discerned by the levels of Cyclin A (S/G2 phase), Cyclin B1 (G2/M transition), and pRb (G1/S transition phase) [19]. Consistent with the heatmap and histogram analyses (Fig. 2), this group decreased with time following treatment with either drug (Fig. 3a, c, d, f). Thus, FLOW-MAP visualizations identified three cell states and intermediate nodes among them, suggestive of different survival or death trajectories.

\section{Machine-learning approaches with CyTOF data identify determinants of cell survival}

To identify features that distinguish viable from apoptotic cells following drug treatment in an unbiased manner, we developed single-cell classification RFMs. The models were developed using a "training dataset" that consisted of $80 \%$ of the data from one of our three treatment replicates ( $80 \%$ Run 3 data). We classified cells from the last timepoints $(24 \mathrm{~h}$ for bortezomib and $72 \mathrm{~h}$ for dexamethasone) into two possible outcomes: "viable" $\left(\mathrm{aCASP}^{\text {neg }}\right)$ or "apoptotic" (aCASP3 $\left.{ }^{\mathrm{pos}}\right)$ (Fig. 4a, S2a). The RFM utilizes an ensemble of decision trees, whereby each tree consists of a series of decisions aiming to accurately sort each cell into a "viable" or "apoptotic" class. Each decision is based on the expression levels of a single marker from the CyTOF data (excluding definitive apoptosis markers, aCASP3 or cPARP) (Fig. 4a, S2a), analogous to a series of hierarchical-gating steps on one marker at a time to arrive at a pure population of interest. In the first model, termed the "BCL-2 family" model, decisions were based on sequential cut-offs of MCL-1, BCL-2, BCL-XL, BIM, BAX, and aBAK levels per cell. In the second "most features" model, pAKT, p53, pSTAT5, MYC IкB $\alpha$, pS6, pCREB, p-p38, and pERK were added to the BCL-2 family proteins. The RFM produces a final classification based on popular vote across many decision trees (Fig. 4a, S2a). The best model was selected through tenfold cross-validation on the training dataset. The relative effectiveness of each marker in separating the two classes was ranked according to the mean decrease in the Gini impurity index across all trees in the ensemble (Fig. 4a). This index reflects how accurate the groupings are (i.e., to what extent the "apoptotic" group might contain "impurities" (viable cells)), assessed by the aCASP 3 status. The parameter that is most discriminating between "apoptotic" versus "viable" cells would provide the greatest reduction in impurity index. 


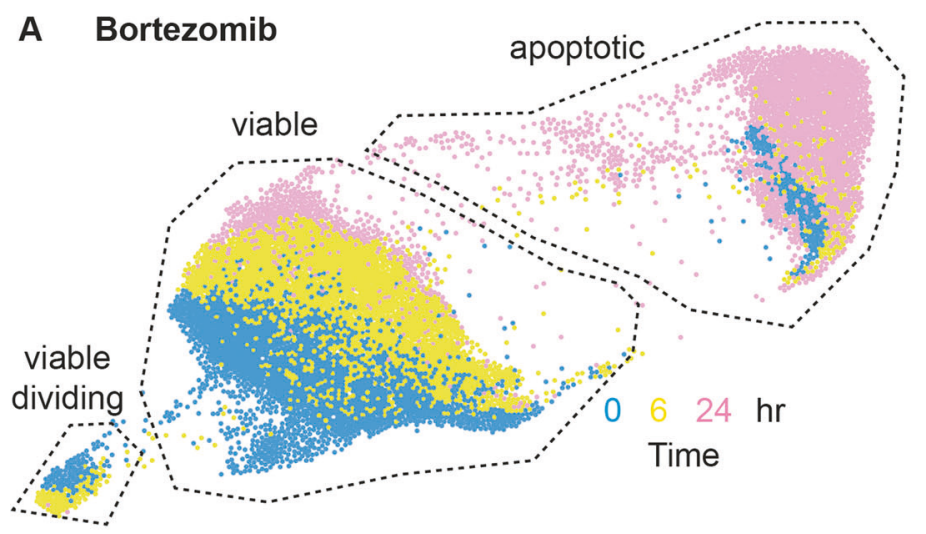

B Bortezomib

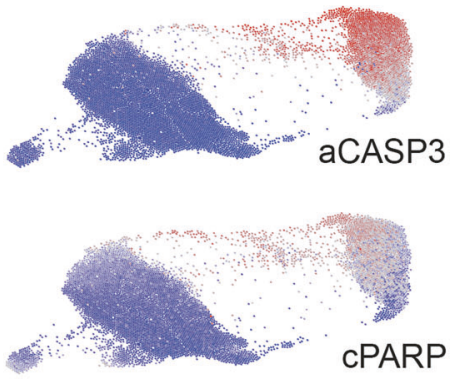

Low $\rightleftharpoons$ High

\section{Bortezomib}

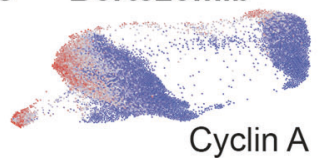

D Dexamethasone
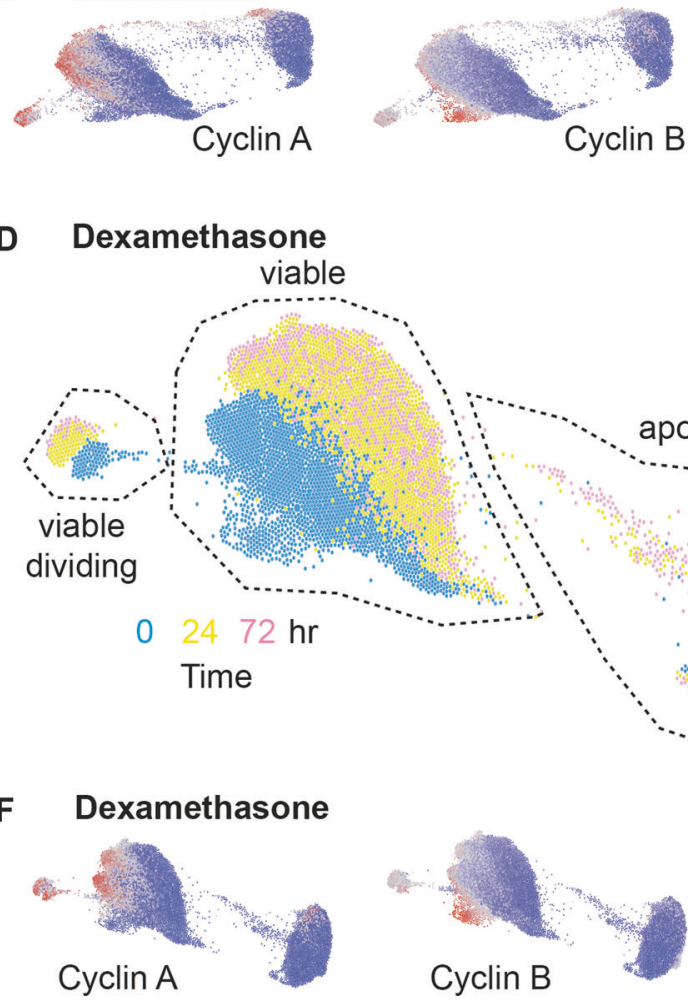

Cyclin B
$\mathbf{F}$

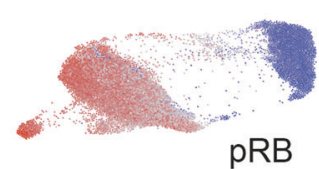

pRB

$\mathrm{pH} 3$

E Dexamethasone
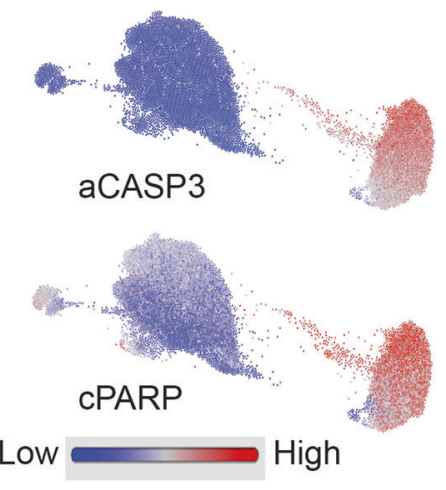

Fig. 3 FLOW-MAP visualizations demonstrate the cell state dynamics of multiple myeloma cells following cytotoxic drug treatment. a FLOW-MAP visualization of the response of live (i.e., cisplatin $^{\text {neg }}$ ) MM.1S cells to bortezomib treatment at 0,6 , and $24 \mathrm{~h}$, colored according to timepoint, or $\mathbf{b}$ expression of activated Caspase-3 (aCASP3) and cleaved PARP (cPARP), or c Cyclin A, Cyclin B, pH3,

In "most features" RFMs, pCREB, and MCL-1 were clearly the most informative markers for distinguishing apoptotic from viable myeloma cells following exposure to either bortezomib or dexamethasone (Fig. 4b). There were also some drug-specific features. For bortezomib treated myeloma cells, p-p38, BIM, and p53 levels were ranked highly, while in dexamethasone-treated cells, IкB $\alpha, \mathrm{pS} 6$, and BIM were also discriminating (Fig. 4b). RFMs trained using the "BCL-2 family" parameter set reinforced the finding that MCL-1 was the most informative feature

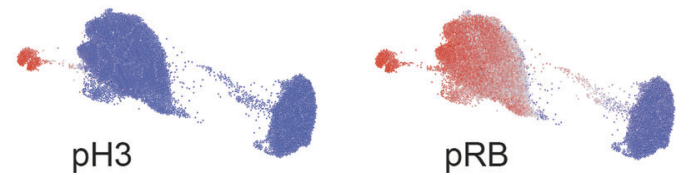

and pRB. d FLOW-MAP visualization of the response of MM.1S cells to dexamethasone treatment at 0,24 , and $72 \mathrm{~h}$, colored according to timepoint, or e expression of activated Caspase-3 (aCASP3) and cleaved PARP (cPARP), or $\mathbf{f}$ Cyclin A, Cyclin $\mathrm{B}, \mathrm{pH} 3$, and $\mathrm{pRB}$. FLOW-MAPs used 10,000 cells randomly subsampled and merged into 2000 clusters from each timepoint of treatment.

distinguishing viable and apoptotic myeloma cell populations (Fig. 4c).

To determine whether the RFMs derived from our training dataset were robust across multiple experiments, we tested the models against "test datasets"; $20 \%$ of Run 3 and two complete, independent technical replicates (Runs 1 and 2). The model predictions of each cell being apoptotic or viable were compared with its actual state based on the levels of aCASP3 (Figs. 4d, S2a). Receiving operating characteristic (ROC) curves had area under the curve 
A

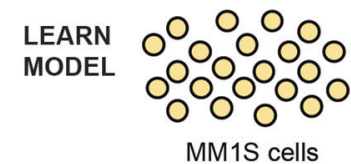

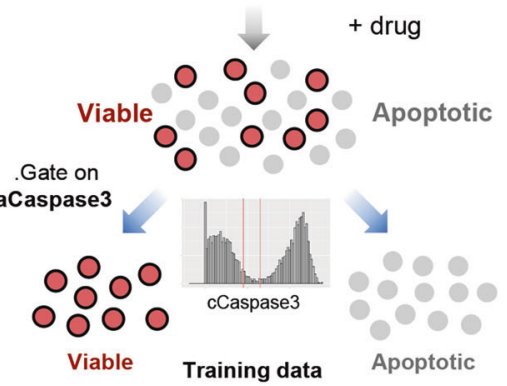

Learn Decision Trees to separate "Viable/Apoptotic"

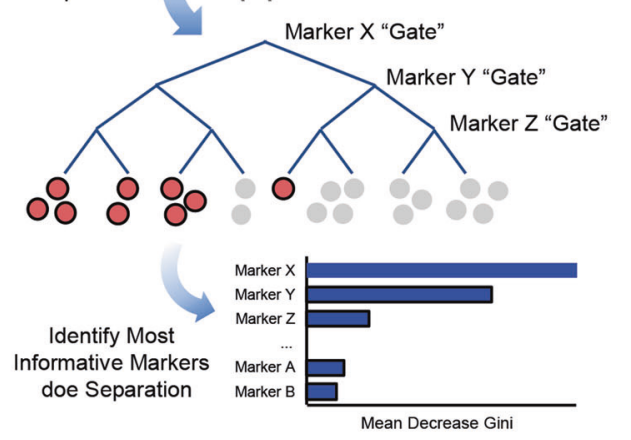

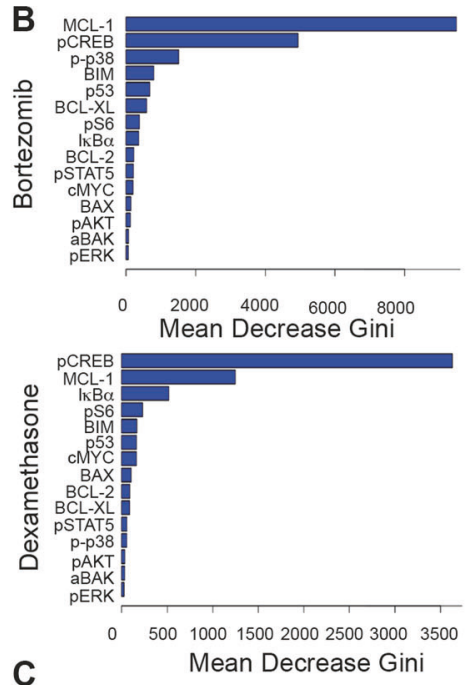

C

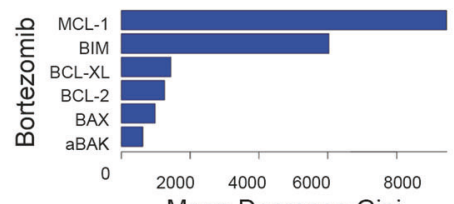

Mean Decrease Gini

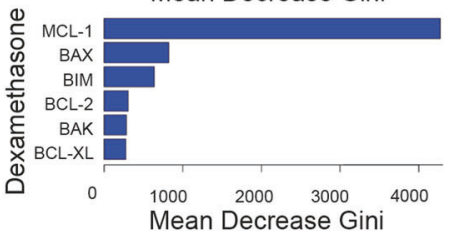

D TEST MODEL

Independent Runs
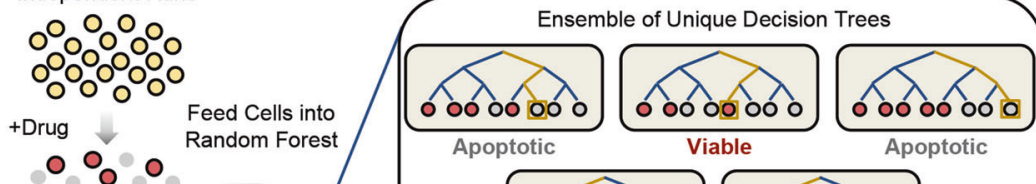
Apoptotic
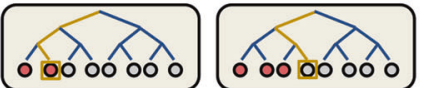

o oo oo oó o ó oóó oó o Apoptotic

True Status

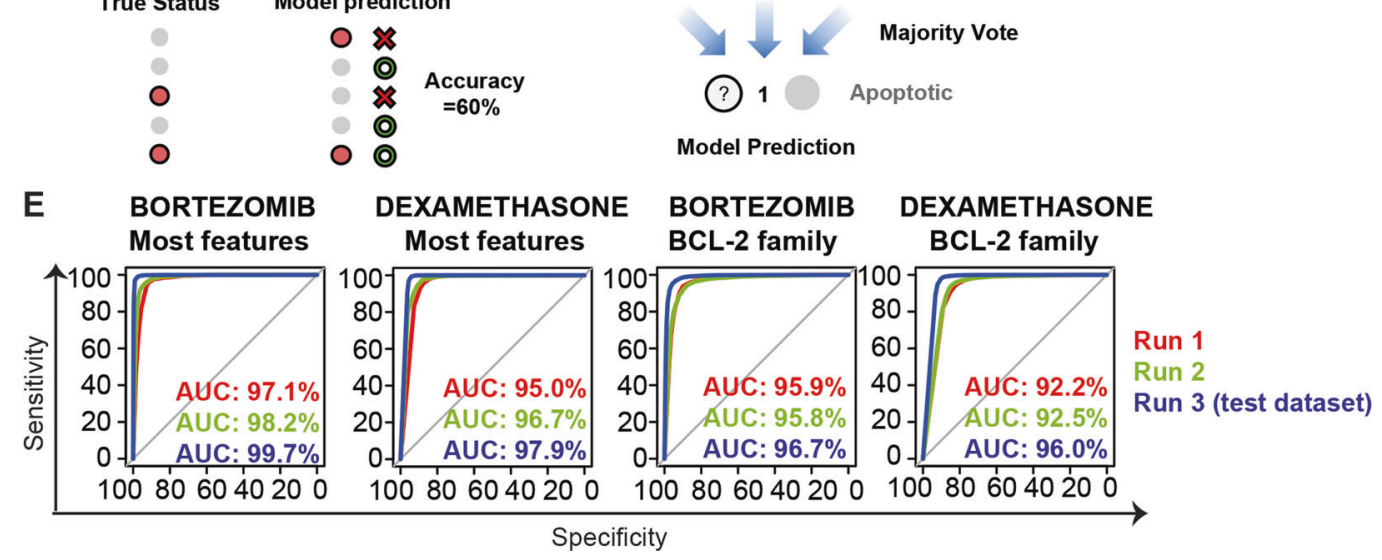

Model prediction
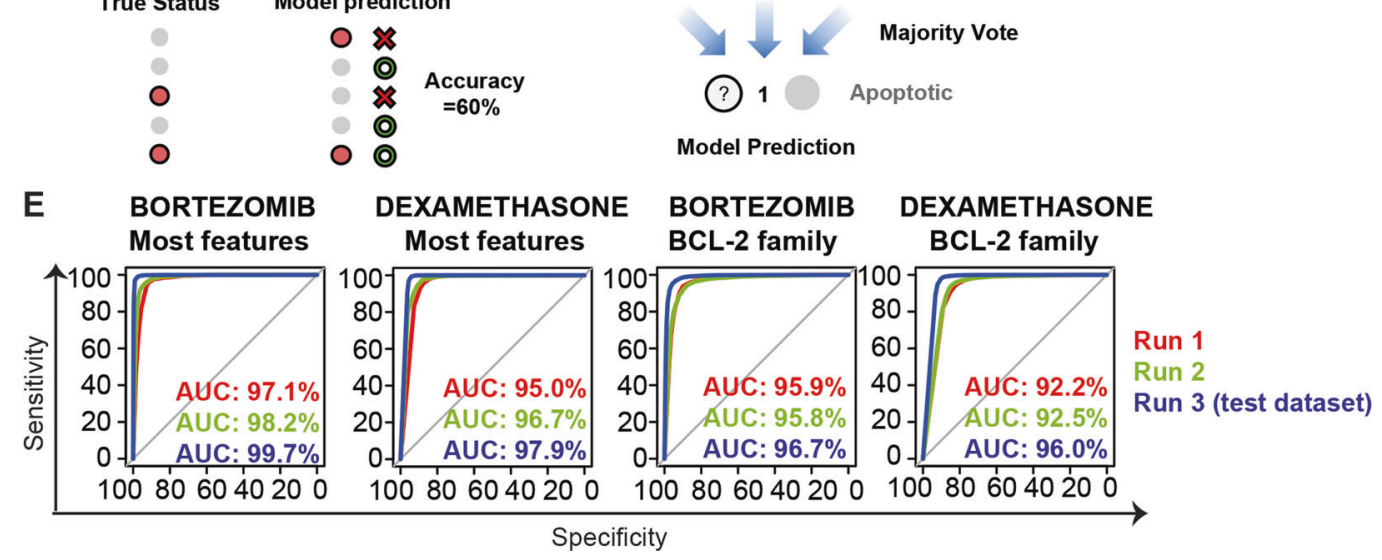

Viable

(AUC) values that exceeded $96 \%$ for all models, indicating that the models are highly sensitive, specific and robust, despite any experimental variations (Fig. 4e). Performance was comparable between the bortezomib- and dexamethasone-treatment models. The "BCL-2 family" model utilizing only the six BCL-2 family proteins as 
Fig. 4 Random forest models trained on mass cytometry time course data identify the key features of cytotoxic drug-induced apoptosis. a Schematic representation of random forest modellearning using single-cell CyTOF data. MM.1S cells at late timepoints after drug treatment are separated into "apoptotic" or "viable" cells based on aCASP3 levels. Random forest models are trained to correctly classify cells into these two groups based on other features, through tenfold cross-validation on training data. Markers that consistently improve the purity of the two populations when used are reflected by a larger mean decrease in Gini impurity index. b Mean decreases in Gini impurity index for all markers included in random forest models, using "most features" measured by CyTOF in single cells, in the bortezomib-specific and dexamethasone-specific models. c Mean decreases in Gini impurity index for "BCL-2 family" proteins included in random forest models for bortezomib- or dexamethasonetreated multiple myeloma cells. d Schematic diagram of the assessment of random forest models using set-aside test data or data from independent CyTOF analyses. Similar to the data used to train the models, MM.1S cells from the last timepoint of drug treatment are gated on aCASP3 levels and designated as "viable" or "apoptotic." The drug-specific random forest models are then tested against these states and accuracy is assessed across the cell population. e Receiving operating characteristic (ROC) curves illustrating the accuracy of each drug-specific model (bortezomib or dexamethasone) using "most features" or restricted to only the "BCL-2 family" parameters. Results are shown for two independent runs (technical replicates Run 1 in red and Run 2 in green) as well as the set-aside test data (Run 3 in blue). The area under the curve (AUC) is shown for each model and each separate dataset.

features was only marginally less accurate than the "most features" model across all datasets (Fig. 4e). These findings indicate that the RFMs are highly accurate and robust, identifying that reduced amounts of MCL-1 within individual myeloma cells treated with cytotoxic drugs may be highly predictive of apoptosis.

\section{Identification of apoptotic trajectories in myeloma cells following drug treatment}

To visualize the features identified by RFM as decisive in the apoptotic response of myeloma cells treated with dexamethasone and/or bortezomib, we produced a single FLOW-MAP incorporating CyTOF data from both time courses (Fig. 5a, b). By filtering the FLOW-MAP graph to show only one treatment time course at a time, we could directly compare features present in bortezomib- versus dexamethasone-treated cells (Fig. 5a, b). Overall, FLOWMAPs of the two treatments overlapped substantially (Fig. 5a, b). This outcome suggests that both drugs induced a similar trajectory to apoptosis in myeloma cells. Consistent with this finding, RFMs trained on data from cells treated with bortezomib or dexamethasone could predict the apoptotic response of cells treated with the other drug with relatively high accuracy (AUC $>80 \%$ ) (Fig. 5c).

Overlay of pCREB and MCL-1 levels on these FLOWMAPs demonstrated higher amounts of these species in viable cells relative to their apoptotic counterparts after treatment with either drug (Fig. 5a, b). Interestingly, cells with low amounts of MCL-1 and pCREB were apparent in nonapoptotic $\left(\mathrm{aCasp}^{\text {neg }}\right)$ population on the FLOW-MAPs (FP1; aCASP $3^{\text {neg }}$ pCREB $^{\text {low }}$ MCL- $\left.{ }^{\text {low }}\right)$, revealing a putative common trajectory to apoptosis provoked by both drugs (Fig. 5a, b). However, an alternative population (FP2; p5 $3^{\text {high }}$ BIM $^{\text {high }}$ MCL-1 ${ }^{\text {high }}$ ) was unique to bortezomib treatment (Fig. 5a). This population accounted for the relatively higher discrimination provided by $\mathrm{p} 53$ in the bortezomib RFM than observed with dexamethasone treatment (Fig. 4b) and suggests an additional apoptotic trajectory in cells treated with the proteasome inhibitor.

\section{Loss of MCL-1 precedes apoptosis in dexamethasone-treated myeloma cells}

We next sought to determine whether the apoptotic trajectory identified by reduced MCL-1 and pCREB (FP1) causes apoptosis or is a consequence of death in other cells. We performed CyTOF analysis of dexamethasone-treated wildtype or $B A K^{-1-} B A X^{-1-}$ MM.1S cells to inhibit apoptosis downstream of MCL-1 pro-survival function. As expected, the aCASP $3^{\text {pos }}{ } \mathrm{CPARP}^{\text {pos }}$ apoptotic population was absent from dexamethasone-treated $B A K^{-1-} B A X^{-1-}$ myeloma cells (Fig. 6a-c), confirming that all of the killing observed in these cells operated via the intrinsic apoptotic pathway. Overlaying the resulting FLOW-MAP graph with the expression of key features showed that FP1 (highlighted by dashed ellipse, right panels) was maintained in $B A K^{-1-} B A X^{-1-}$ cells, despite not progressing to the final stages of apoptosis (Fig. 6a, b). These data indicate that the features identified by the RFMs occur in the same cells and are not a consequence of BAX/BAKmediated apoptosis following dexamethasone treatment, but rather, likely to be the critical initiating event.

\section{MCL-1 inhibition synergizes with dexamethasone to induce apoptosis in myeloma cells}

Our data show that high levels of pCREB and MCL-1 coincide in viable cells and discriminate them from cells undergoing dexamethasone-induced apoptosis. CREB can control Mcll transcription [34]; therefore, we hypothesized that selective inhibition of MCL-1 alone would enhance the apoptotic response of myeloma cells to dexamethasone. To test this hypothesis, we compared apoptosis in MM.1S cells using dexamethasone combined with the BCL-2 inhibitor, ABT-199 [35], the BCL-XL inhibitor, A-1331852 [8], or the MCL-1 inhibitor, S63845 [7]. Limited apoptosis was induced by dexamethasone treatment combined with BCL-2 inhibition for $24 \mathrm{~h}$ (Fig. 7a). The BCL-XL inhibitor alone induced substantial apoptosis at high doses $(>0.1 \mu \mathrm{M})$ and some synergistic killing when combined with dexamethasone (Fig. 7a). However, the combination of 

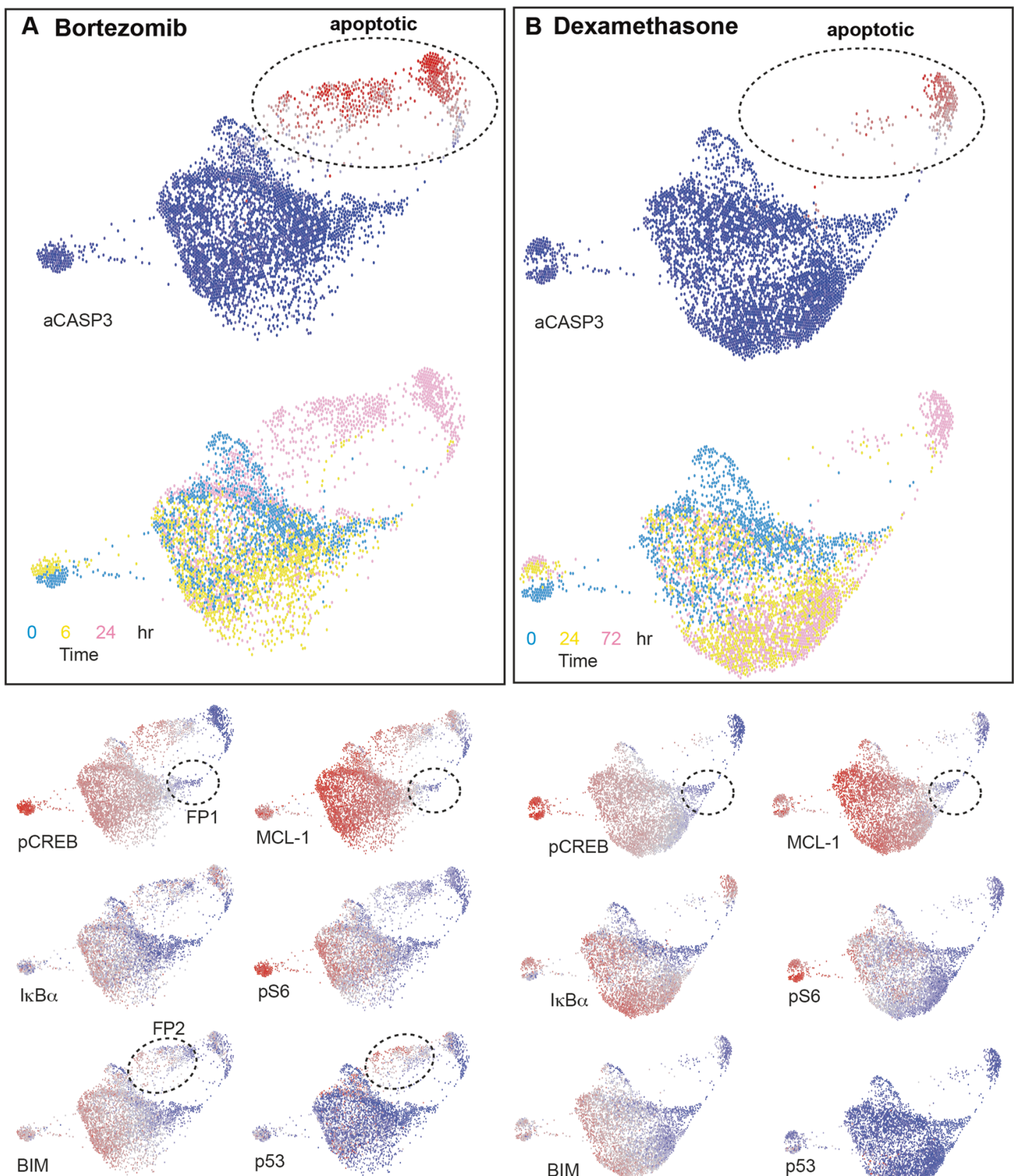

Feature population (FP) 1: aCASP3 $3^{\text {low }}$ pCREB low MCL-1 10w Feature population (FP) 2: $p 53^{\text {high }}$ BIM ${ }^{\text {high }} \mathrm{MCL}-1^{\text {high }}$

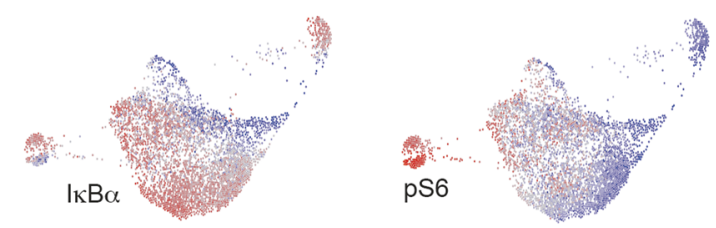

C

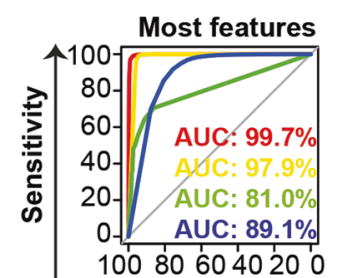

BCL-2 family

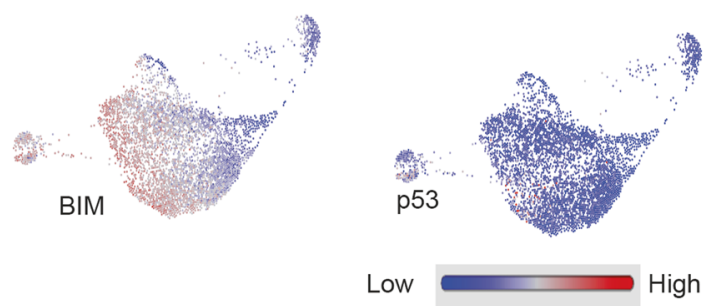

Specificity

dexamethasone and the MCL-1 inhibitor S63845 caused marked cell death at low concentrations, with $1.25 \mu \mathrm{M}$ dexamethasone and $0.02 \mu \mathrm{M}$ S63845 inducing apoptosis in
$90 \%$ of MM.1S cells (Fig. 7a, b). The interaction landscape for the drug combinations [36] indicated synergism between dexamethasone and the MCL-1 inhibitor over the whole 
Fig. 5 FLOW-MAP comparison of key model features following bortezomib or dexamethasone treatment. FLOW-MAP visualization comparing response with (a) bortezomib or (b) dexamethasone treatment in MM.1S cells. A single FLOW-MAP was produced with data from both treatment time courses using the same markers as clustering variables as listed in Fig. 3, colored by time and levels of aCASP3, pCREB, MCL-1, IкB $\alpha$, pS6, BIM, and p53. c ROC curve showing the accuracy of cross-testing drug-specific models, with random forest models using "most features" or restricted to only the "BCL-2 family" proteins. The AUC is shown for each model and each pair of drug-specific model and drug data.

dose-response matrix, with the strongest effect (BLISS scores $\sim 50-60$ ) found when the dose of S63845 was fixed at $0.156 \mu \mathrm{M}$ (Fig. 7b). All combinations of BH3-mimetic drugs with dexamethasone achieved some level of cooperativity in cell killing at the highest drug concentration tested $(10 \mu \mathrm{M})$; however, the highest delta BLISS score and cell death was observed in the dexamethasone/S63845 combination (Fig. 7b). The sensitivity of MM.1S cells to this drug combination was also observed to some extent in a xenograft model (Supplementary Fig. 3a, b). Furthermore, the combination of $\mathrm{S} 63845$ with bortezomib induced greater apoptosis of MM.1S cells than either drug alone, although the relatively high levels of cell death induced by bortezomib limited the capacity to resolve further synergy (Supplementary Fig. 3c). Hence, these comparisons of the synergism of $\mathrm{BH} 3$-mimetic drugs with dexamethasone or bortezomib were consistent with the RFM ranking of MCL1 as the pivotal pro-survival protein following treatment (Fig. 4).

To determine whether this synergy between dexamethasone and the MCL-1 inhibitor S63845 was a common feature in myeloma cells, we also generated interaction landscapes for five other cell lines following treatment with drugs for $24 \mathrm{~h}$. We found that dexamethasone and S63845 induced synergistic killing of OPM2 and AMO1 cells but had minimal or no synergy in KMS-12-BM, U266, and H929 cells (Fig. 7c).

These findings were recapitulated in primary myeloma cells. Cells from bone marrow samples isolated from 12 different MM patients at diagnosis were incubated for $24 \mathrm{~h}$ with $100 \mathrm{nM}$ dexamethasone and/or $100 \mathrm{nM}$ S63845 and apoptosis was quantified relative to the level of spontaneous death observed in vehicle-treated cells. Consistent with the MM.1S cell line data, synergistic killing with dexamethasone and S63845 was observed in primary myeloma cells from 10 of 12 of patients, with four patients displaying very strong synergy (BLISS scores $>16$ ) (Fig. 8a, b). These results indicate that combining an MCL-1 inhibitor with dexamethasone has the potential to enhance killing myeloma cells, yet that there is also substantial heterogeneity in the response. To probe this observation further and test the capacity for mass cytometry to detect cell survival parameters of primary patient cells, we subjected two samples to
CyTOF analysis with an expanded panel of probes, designed to resolve myeloma cells. Visualization of these data using t-distributed stochastic neighbor embedding (tSNE), with overlay of the expression of key markers, resolved a putative myeloma cell population defined as $\mathrm{CD} 45^{\text {low }} \mathrm{CD} 38^{\text {high }} \mathrm{CD} 138^{\text {pos/neg }}$ and IRF4 ${ }^{\text {pos }}$ in bone marrow cells from both patients (Fig. 8c). Interestingly, the amounts of MCL-1 in these myeloma cell populations differed. Relatively high amounts were observed in myeloma cells from patient \#12, which exhibited strong synergistic killing by dexamethasone and S63845, compared with those from patient \#11, in which this drug combination low synergy (Fig. 8b, d). These data demonstrate the capacity of CyTOF to resolve myeloma cells in bone marrow samples from patients and their expression of key pro-survival proteins.

\section{Discussion}

The importance of the BCL-2 protein family in the control of cell survival makes them attractive drug targets in MM. The BH3-mimetic drugs navitoclax (inhibitor of BCL-2, BCL-XL, and BCL-W) and venetoclax (a specific inhibitor of BCL-2) can induce apoptosis in primary patient-derived myeloma cells in vitro and ex vivo $[16,17,37-40]$. Accordingly, venetoclax monotherapy in patients with relapsed/refractory MM has demonstrated efficacy, but thus far has yielded only a modest overall response (21\%) relative to chronic lymphocytic leukemia or mantle cell lymphoma [9, 18]. Although this outcome highlights the potential for BH3-mimetic drugs in treating $\mathrm{MM}$, it also demonstrates that heterogeneity among MM patients and therapeutic resistance are important problems. Studies have shown that myeloma cells with the $\mathrm{t}(11 ; 14)$ chromosomal translocation exhibit heightened responses to venetoclax [38, 41], with evidence that these cancerous cells have higher expression of BCL-2 relative to BCL-XL and MCL1 [18]. Coadministration of standard-of-care therapies, such as dexamethasone, may also change this interaction landscape. Heterogeneous responses to dexamethasone can alter the balance of the BCL-2 family of proteins at the population level in myeloma cells $[42,43]$. The approaches in our study could be used to monitor the relative expression of BCL-2 family members and other pathways at the singlecell level in myeloma cells from patients at diagnosis and during therapy to inform optimal therapeutic combinations, including $\mathrm{BH} 3$-mimetic drugs.

It is clear that the interplay amongst members of the BCL-2 family of proteins can engender resistance to navitoclax or venetoclax (e.g., [44-46]). However, it has been challenging to determine the critical events in the small fraction of cells that, at any point in time, are engaged in making the decision to live or die. Mass cytometry is well 

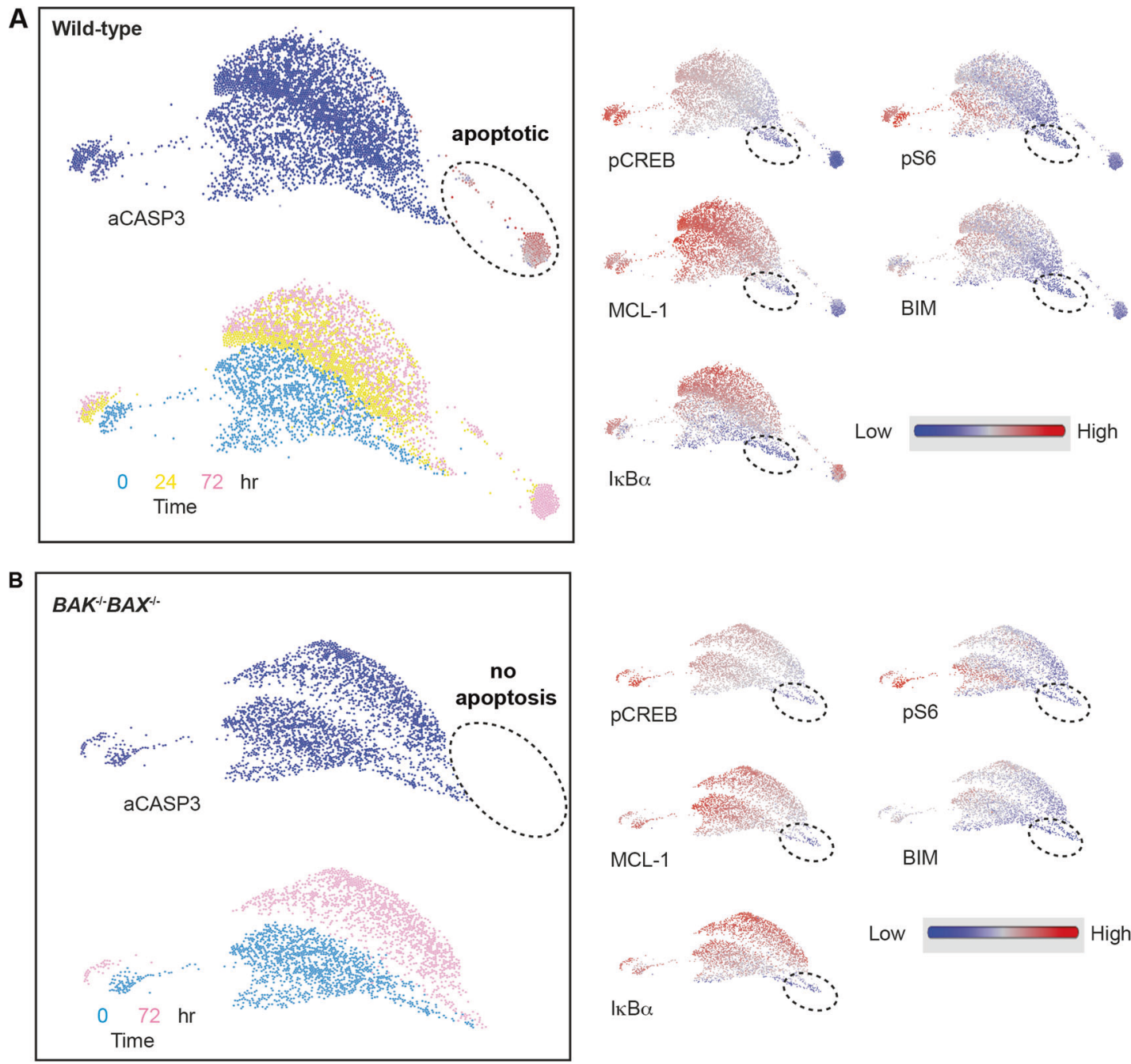

MCL-1
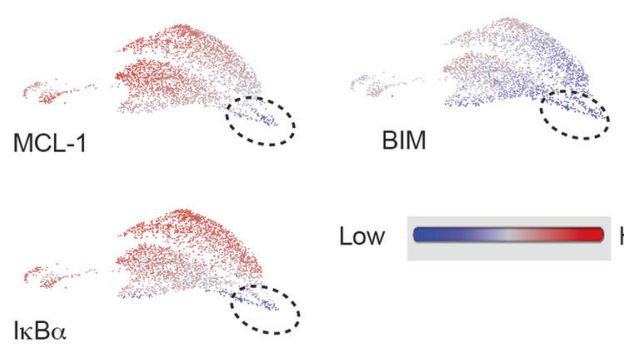

Low

$\mathrm{I} \mathrm{B} \alpha$

C

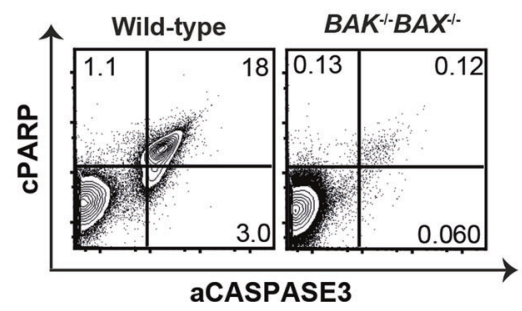

Fig. 6 FLOW-MAP visualization of the key features in the apoptotic response to dexamethasone in multiple myeloma cells reveals a transitional population. FLOW-MAP visualization of response to dexamethasone treatment comparing (a) parental MM.1 S and (b) $B A K^{-1-} B A X^{-1-}$ MM.1S cell line. A single FLOW-MAP was produced with data from both cell lines, using 6000 cells randomly subsampled

suited to this endeavor because it allows high-throughput, high-parameter resolution at the single-cell level. Our validated panel of antibodies specific for the major apoptotic regulators and key-signaling states could resolve the pivotal parameters in myeloma cell apoptosis induced by dexamethasone or bortezomib. Machine-learning approaches and visualization algorithms that interrogate the data at the and merged into 1200 clusters from each timepoint of treatment in each cell line. The FLOW-MAP was constructed using the same markers as clustering variables as listed in Fig. 3, and colored according to time or levels of aCASP3, pCREB, pS6, MCL-1, BIM, and IкB $\alpha$. c Dot plot of cPARP and aCASP3 levels for WT MM.1S and $B A K^{-1-} B A X^{-1-}$ MM.1S cells.

single-cell level, rather than analysis of summaries of population behavior (e.g., mean or aggregate expression levels), were most revealing in this regard. The utility of this analytical paradigm was demonstrated by the identification and exploitation of a pCREB/MCL-1 axis that maintains the survival of myeloma cells following dexamethasone or bortezomib treatment. 
A

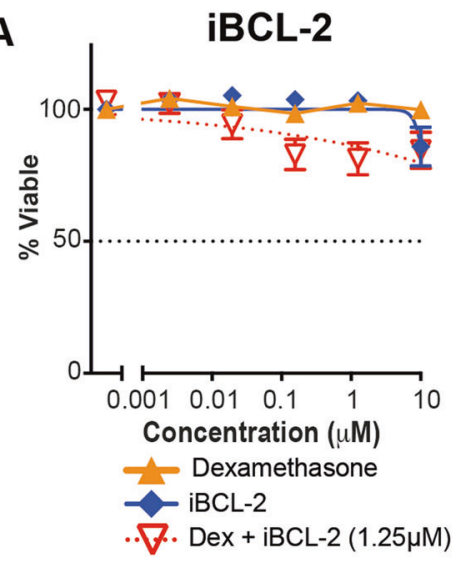

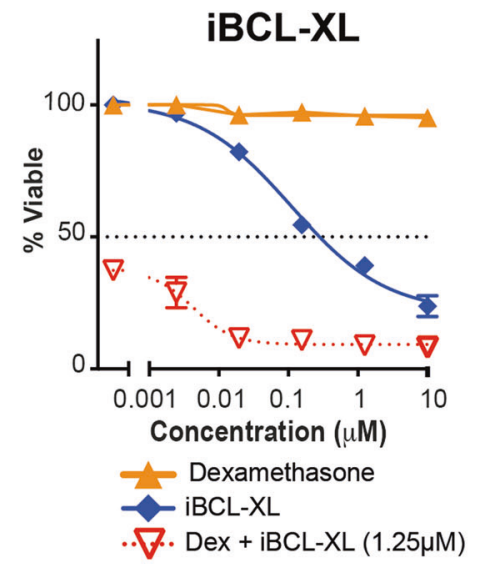

iMCL-1

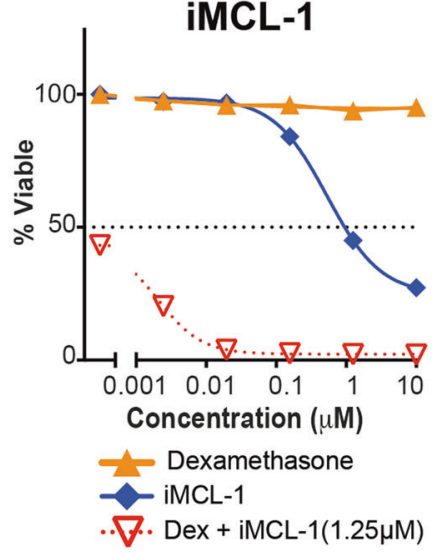

B

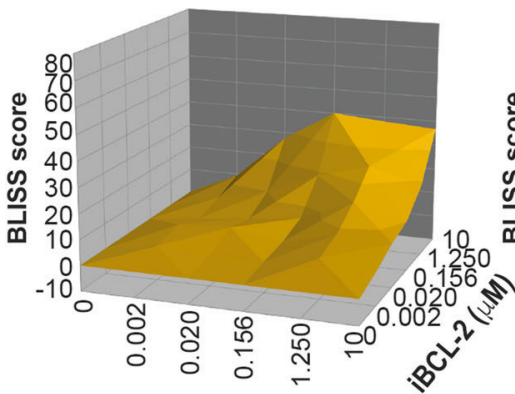

Dexamethasone ( $\mu \mathrm{M})$
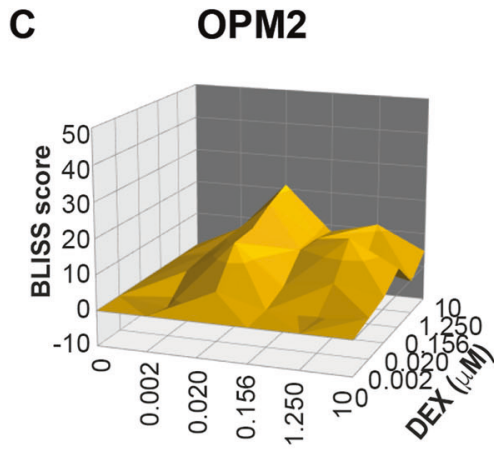

iMCL-1 (uM)

KMS-12-BM

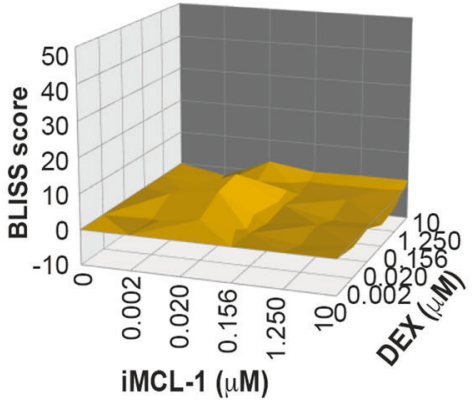

iBCL-XL

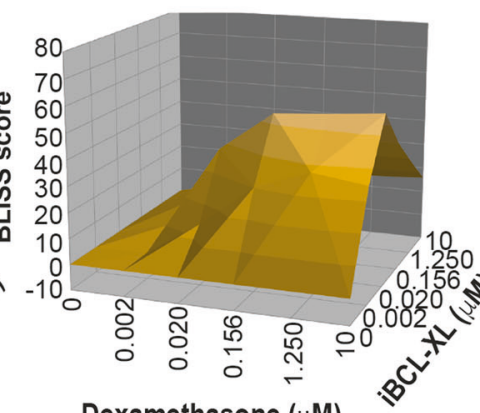

Dexamethasone $(\mu \mathrm{M})$

AMO1

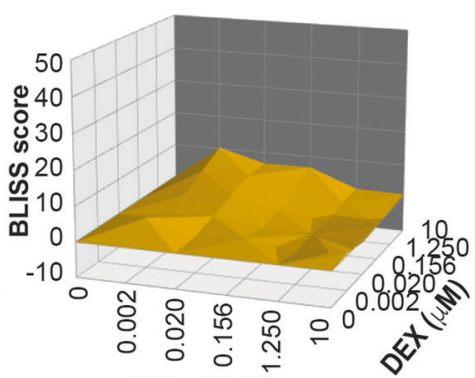

iMCL-1 (uM)

\section{H929}

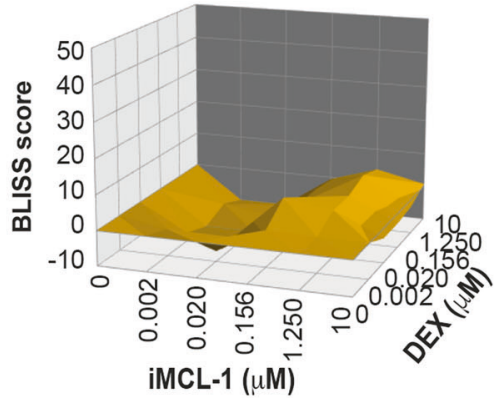

- 50-60

- 40-50

- 30-40

- 20-30

- 10-20

$0-10$

- $-10-0$
BCL-2, BCL-XL, or MCL-1 have each been implicated in myeloma cell survival and some studies have employed putative $\mathrm{BH} 3$ mimetics in an effort to target these proteins.
However, some presumptive BH3-mimetic compounds do not specifically kill via the intrinsic pathway but induce cell death in an entirely nonspecific manner (e.g., [47]), 
Fig. 7 Synergistic killing of multiple myeloma cells with MCL-1 inhibition combined with dexamethasone. a Mean $( \pm$ SEM) viability of the MM.1S cell line as measured by flow cytometric analysis of propidium iodide $(\mathrm{PI})$ versus Annexin $\mathrm{V}$ staining following treatment with the combination of $1.25 \mu \mathrm{M}$ of $\mathrm{BH} 3$-mimetic drugs as inhibitors

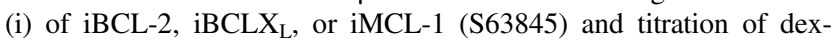
amethasone $(0-10 \mu \mathrm{M})$ compared with each treatment alone for $24 \mathrm{~h}$. The dotted horizontal line represents $50 \%$ loss of viability. b The interaction landscapes identified from combination treatments in $\mathbf{a}$. For each combination, the landscapes are shown in $3 \mathrm{D}$ where the BLISS scores represent the excess percentage inhibition beyond that expected from additive interaction. c The interaction landscapes of OPM2, AMO1, U266, KMS-12-BM, and H929 cell lines after combination treatments with S63845 and dexamethasone for $24 \mathrm{~h}$. Data from a and $\mathbf{b}$ are representative of three independent experiments. Data from $\mathbf{c}$ are representative of two independent experiments.

therefore the utility of pharmacological targeting these prosurvival proteins in myeloma remains an important question. We recently reported that MCL-1 was critical in many common myeloma cell lines using gene editing and $\mathrm{BH} 3$ peptide variants [15] or validated MCL-1-specific BH3 mimetics [7]. Furthermore, MCL-1 is highly expressed in primary myeloma cells from patients and higher levels of expression were associated with a propensity to early relapse and shorter patient survival with standard therapy [48]. Our finding that the MCL-1 inhibitor, S63845, synergized with dexamethasone to kill myeloma cells extends upon these studies and highlights the importance of this pro-survival protein in determining response versus resistance to dexamethasone. A direct mechanism is likely, whereby glucocorticoid receptor activation inhibits CREB activity [49], which in turn curtails Mcll transcription [50] and diminishes the amounts of this short-lived pro-survival protein to induce apoptosis via the intrinsic pathway. Why only a fraction of the cells exposed to dexamethasone activate this cascade at any point in time remains an interesting question. A deeper understanding of how these mechanisms operate in individual cells may guide dosing regimens for the combination of dexamethasone with MCL1 inhibitors, and the concentrations of the latter that best balance efficacy and safety.

A

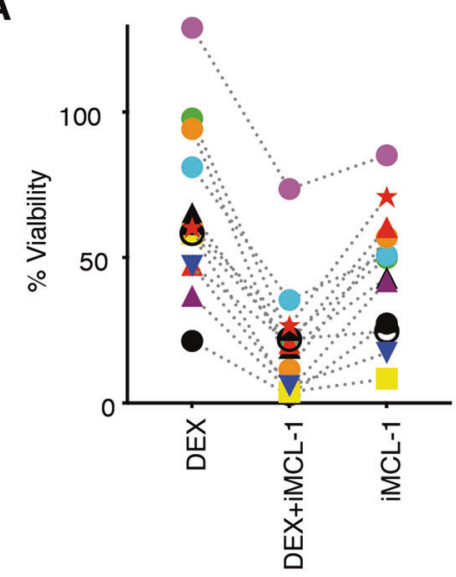

C
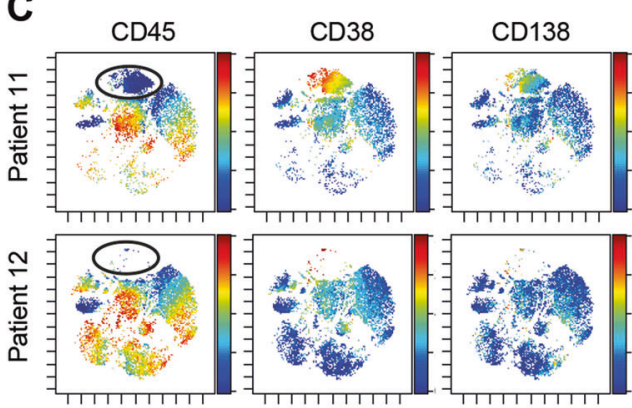

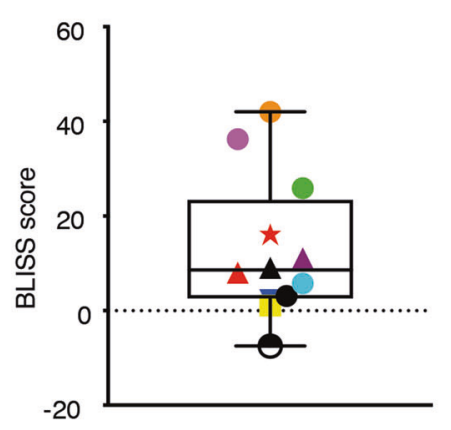

IRF4

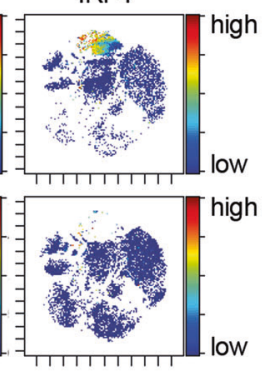

D

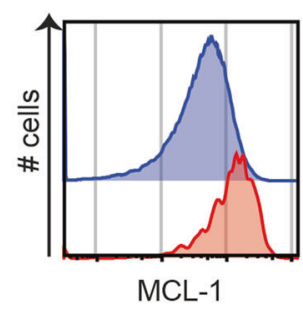

Geo mean | BLISS

Patient 11: $2.9 \mid 2.3$

Patient 12: $10.6 \mid 16.1$
Fig. 8 Synergistic killing of myeloma cells from patients by combining MCL-1 inhibition with dexamethasone. a Viability of bone marrow cells isolated from multiple myeloma patients assessed after $24 \mathrm{~h}$ of treatment with dexamethasone, the combination of dexamethasone and S63845, and S63845 alone, relative to DMSO control. b Box and whisker plot showing BLISS scores for ex vivo drug synergy in primary myeloma cells from patients. $\mathbf{c}$ tSNE analysis of treatment naive multiple myeloma patients 11 and 12 to represent the higher dimensional relationships among bone marrow cells. Ellipse highlights putative myeloma populations with the phenotype $\mathrm{CD} 45^{\text {low }} \mathrm{CD} 38^{\text {pos }} \mathrm{CD} 138^{\text {pos/neg }} \mathrm{IRF} 4^{\text {pos }}$. d MCL-1 protein levels measured by CyTOF gated on $\mathrm{CD} 45^{\text {low }} \mathrm{CD} 38^{\text {high }} \mathrm{CD} 138^{\text {pos/neg }}$ and $\mathrm{IRF} 4{ }^{\text {pos }}$ treatment naive myeloma cells from patients 11 and 12 . 
Beyond mechanistic studies of cell death, the mass cytometry panel reported here may also be useful for profiling cancer cell subpopulations in patients to identify predictors of responses to treatment, particularly with BH3mimetic drugs. Previous mass cytometric analysis of MM with cell surface markers delineated the aberrant B cell phenotype in detail and identified substantial heterogeneity among patients [34]. Similarly, a study in chronic myeloid leukemia also incorporated CyTOF profiling of some BCL2 family proteins and found high expression of BCL-2 in a population with stem cell-like characteristics that could be targeted with venetoclax in preclinical models and primary patient samples [51]. The ability to identify and characterize subpopulations is especially valuable in MM as intraclonal heterogeneity has gained significant interest, whereby different clones within an individual patient can predominate depending on the pressure exerted by therapeutic interventions [52]. Our preliminary data in two patients demonstrates that an expanded CyTOF panel built around our probes for cell death proteins can resolve functionally relevant changes in myeloma cell heterogeneity. The capacity to follow the response of myeloma cells from patients following treatment with standard-of-care therapies and/or BH3-mimetic drugs may identify predictors of treatment response and mechanisms of resistance that could influence therapy; an approach that has had value in other settings $[45,46]$.

Taken together, these findings provide a strong rationale for further investigation of dexamethasone and MCL-1 inhibitor combinations in the clinic. Several compounds, including S64315 (a derivative of S63845 used in this study [7] and AMG $176[53,54])$ are now under investigation in early phase clinical trials (e.g., ClinicalTrials.gov identifier NCT02992483).

Acknowledgements CET is supported by an Australian NHMRC Early Career Fellowship (1089072) and a Fulbright Australia-America Postdoctoral Fellowship. PLF is supported by a Leukaemia Foundation of Australia Clinical PhD Scholarship. MSYL is supported by a NHMRC/RACP Gus Nossal PhD scholarship (1075151). GPN is supported by the Rachford and Carlotta A. Harris Endowed Chair. DHDG is supported by Australian NHMRC Fellowships (1090236 and 1158024). AS is supported by an Australian NHMRC Senior Principal Research Fellowship (1020363). MEK is supported by the National Cancer Institute of the National Institutes of Health under Award Number F99CA212231 and Stanford University's Diversifying Academia, Recruiting Excellence Fellowship. This work was supported by grants to GPN: U19 AI057229, 1U19AI100627, Department of Defense (CDMRP), Northrop-Grumman Corporation, R01CA184968, 1R33CA183654-01, R33CA183692, R01CA184968, 1R21CA183660, 1R01NS08953304, OPP1113682, 5UH2AR067676, 1R01CA19665701, R01HL120724; grants to DHDG: Cancer Council of Victoria Grants-in-Aid (1146518 and 1102104); grants to AWR/ DCSH: NHRMC (1113577, 1016701, 1079560); grants to AS: Leukemia and Lymphoma Society SCOR 7001-13, NHMRC program grant 1016701. This work was made possible through Victorian State Government Operational Infrastructure Support and Australian
Government NHMRC IRIISS. The authors thank Prof. Silvia Plevritis (Stanford University) for advice and critical discussions, Dr Andrew Mitchell (University of Melbourne) for assistance with mass cytometry maintenance/operation, Chris Riffkin (WEHI) for assistance with cell culture and Assoc. Prof Grant Dewson for assistance with densitometric analysis of western blot data. This work was performed in part at the Materials Characterization and Fabrication Platform (MCFP) at the University of Melbourne and the Victorian Node of the Australian National Fabrication Facility (ANFF) with support from the Victorian Comprehensive Cancer Centre.

Author contributions Conception and design: CET, DCSH, GPN, DHDG, and MEK. Development of methodology: CET and MEK. Acquisition of data (provided animals, acquired and managed patients, provided facilities, etc.): CET, JG, DS, TT, CJV, PLF, MSYL, GG, SJH, AWR, DCSH, and MEK. Analysis and interpretation of data (e.g., statistical analysis, biostatistics, and computational analysis): CET, JG, and MEK. Writing, review, and/or revision of the paper: CET, MEK, DHDG, CJV, PLF, MSYL, AS, AWR, DCSH, and GPN.

\section{Compliance with ethical standards}

Conflict of interest GPN is a paid consultant for Fluidigm, the manufacturer that produced some of the reagents and instrumentation used in this study. CET, JG, DS, TT, CJV, PLF, MSYL, AS, AWR, DCSH, and DHDG are employees of Walter and Eliza Hall Institute of Medical Research which receives milestone and royalty payments related to venetoclax (iBCL-2). SJH has received research funding and has participated in Advisory boards from Janssen Cilag (bortezomib), Abbvie (venetoclax), and Amgen (iMCL-1). Researchers at the Walter and Eliza Hall Institute of Medical Research in the Strasser, Roberts, Huang, and Gray laboratories collaborate with Servier on the development of MCL-1 inhibitors. All other authors declare no competing financial interests.

Publisher's note Springer Nature remains neutral with regard to jurisdictional claims in published maps and institutional affiliations.

\section{References}

1. Hanahan D, Weinberg RA. Hallmarks of cancer: the next generation. Cell. 2011;144:646-74.

2. Delbridge AR, Strasser A. The BCL-2 protein family, BH3mimetics and cancer therapy. Cell Death Differ. 2015;22: $1071-80$

3. Green DR, Reed JC. Mitochondria and apoptosis. Science. 1998;281:1309-12.

4. Tait SW, Green DR. Mitochondria and cell death: outer membrane permeabilization and beyond. Nat Rev Mol Cell Biol. 2010;11: 621-32.

5. Korsmeyer SJ, Wei MC, Saito M, Weiler S, Oh KJ, Schlesinger PH. Pro-apoptotic cascade activates BID, which oligomerizes BAK or BAX into pores that result in the release of cytochrome c. Cell Death Differ. 2000;7:1166-73.

6. Luo X, Budihardjo I, Zou H, Slaughter C, Wang X. Bid, a Bcl2 interacting protein, mediates cytochrome $\mathrm{c}$ release from mitochondria in response to activation of cell surface death receptors. Cell. 1998;94:481-90.

7. Kotschy A, Szlavik Z, Murray J, Davidson J, Maragno AL, Le Toumelin-Braizat G, et al. The MCL1 inhibitor S63845 is tolerable and effective in diverse cancer models. Nature. 2016;538: 477-82.

8. Leverson JD, Phillips DC, Mitten MJ, Boghaert ER, Diaz D, Tahir SK, et al. Exploiting selective BCL-2 family inhibitors to dissect 
cell survival dependencies and define improved strategies for cancer therapy. Sci Transl Med. 2015;7:279ra240.

9. Roberts AW, Huang D. Targeting BCL2 with BH3 mimetics: basic science and clinical application of venetoclax in chronic lymphocytic leukemia and related B cell malignancies. Clin Pharm Ther. 2017;101:89-98.

10. Kumar SK, Rajkumar V, Kyle RA, van Duin M, Sonneveld P, Mateos MV, et al. Multiple myeloma. Nat Rev Dis Prim. 2017; 3:17046.

11. Braggio E, Kortum KM, Stewart AK. SnapShot: multiple myeloma. Cancer Cell. 2015;28:678.e671.

12. Abdi J, Chen G, Chang H. Drug resistance in multiple myeloma: latest findings and new concepts on molecular mechanisms. Oncotarget. 2013;4:2186-207.

13. Gomez-Bougie P, Wuilleme-Toumi S, Menoret E, Trichet V, Robillard N, Philippe M, et al. Noxa up-regulation and Mcl-1 cleavage are associated to apoptosis induction by bortezomib in multiple myeloma. Cancer Res. 2007;67:5418-24.

14. Podar K, Gouill SL, Zhang J, Opferman JT, Zorn E, Tai YT, et al. A pivotal role for $\mathrm{Mcl}-1$ in Bortezomib-induced apoptosis. Oncogene. 2008;27:721-31.

15. Gong JN, Khong T, Segal D, Yao Y, Riffkin CD, Garnier JM, et al. Hierarchy for targeting prosurvival BCL2 family proteins in multiple myeloma: pivotal role of MCL1. Blood. 2016;128: 1834-44.

16. Bodet L, Gomez-Bougie P, Touzeau C, Dousset C, Descamps G, Maiga S, et al. ABT-737 is highly effective against molecular subgroups of multiple myeloma. Blood. 2011;118:3901-10.

17. Kline MP, Rajkumar SV, Timm MM, Kimlinger TK, Haug JL, Lust JA, et al. ABT-737, an inhibitor of Bcl-2 family proteins, is a potent inducer of apoptosis in multiple myeloma cells. Leukemia. 2007;21:1549-60.

18. Kumar S, Kaufman JL, Gasparetto C, Mikhael J, Vij R, Pegourie $\mathrm{B}$, et al. Efficacy of venetoclax as targeted therapy for relapsed/ refractory $\mathrm{t}(11 ; 14)$ multiple myeloma. Blood. 2017;130:2401-9.

19. Behbehani GK, Bendall SC, Clutter MR, Fantl WJ, Nolan GP. Single-cell mass cytometry adapted to measurements of the cell cycle. Cytom A. 2012;81:552-66.

20. Bendall SC, Simonds EF, Qiu P, Amir el AD, Krutzik PO, Finck $\mathrm{R}$, et al. Single-cell mass cytometry of differential immune and drug responses across a human hematopoietic continuum. Science. 2011;332:687-96.

21. Bodenmiller B, Zunder ER, Finck R, Chen TJ, Savig ES, Bruggner RV, et al. Multiplexed mass cytometry profiling of cellular states perturbed by small-molecule regulators. Nat Biotechnol. 2012;30:858-67.

22. Fienberg HG, Simonds EF, Fantl WJ, Nolan GP, Bodenmiller B. A platinum-based covalent viability reagent for single-cell mass cytometry. Cytom A. 2012;81:467-75.

23. Zunder ER, Lujan E, Goltsev Y, Wernig M, Nolan GP. A continuous molecular roadmap to iPSC reprogramming through progression analysis of single-cell mass cytometry. Cell Stem Cell. 2015;16:323-37.

24. Zunder ER, Finck R, Behbehani GK, Amir el AD, Krishnaswamy $\mathrm{S}$, Gonzalez VD, et al. Palladium-based mass tag cell barcoding with a doublet-filtering scheme and single-cell deconvolution algorithm. Nat Protoc. 2015;10:316-33.

25. Finck R, Simonds EF, Jager A, Krishnaswamy S, Sachs K, Fantl $\mathrm{W}$, et al. Normalization of mass cytometry data with bead standards. Cytom A. 2013;83:483-94.

26. Kotecha N, Krutzik PO, Irish JM. Web-based analysis and publication of flow cytometry experiments. Curr Protoc Cytom. 2010;53:10.17.1-10.17.24.

27. Bliss CI. The toxicity of poisons applied jointly. Ann Appl Biol. 1939;26:585-615.
28. Westphal D, Kluck RM, Dewson G. Building blocks of the apoptotic pore: how Bax and Bak are activated and oligomerize during apoptosis. Cell Death Differ. 2014;21:196-205.

29. Dewson G, Kratina T, Sim HW, Puthalakath H, Adams JM, Colman PM, et al. To trigger apoptosis, Bak exposes its BH3 domain and homodimerizes via $\mathrm{BH} 3$ :groove interactions. Mol Cell. 2008;30:369-80.

30. Kruger FJ. Pilot study of the potential use of leucocyte adherence to Schistosoma haematobium eggs as an indication of immunity to reinfection. Trans R Soc Trop Med Hyg. 1991;85:83.

31. Alsop AE, Fennell SC, Bartolo RC, Tan IK, Dewson G, Kluck RM. Dissociation of Bak alpha1 helix from the core and latch domains is required for apoptosis. Nat Commun. 2015;6:6841.

32. Hideshima T, Chauhan D, Hayashi T, Akiyama M, Mitsiades N, Mitsiades C, et al. Proteasome inhibitor PS-341 abrogates IL-6 triggered signaling cascades via caspase-dependent downregulation of gp130 in multiple myeloma. Oncogene. 2003;22: 8386-93.

33. Hideshima T, Nakamura N, Chauhan D, Anderson KC. Biologic sequelae of interleukin-6 induced PI3-K/Akt signaling in multiple myeloma. Oncogene. 2001;20:5991-6000.

34. Hansmann L, Blum L, Ju CH, Liedtke M, Robinson WH, Davis MM. Mass cytometry analysis shows that a novel memory phenotype B cell is expanded in multiple myeloma. Cancer Immunol Res. 2015;3:650-60.

35. Souers AJ, Leverson JD, Boghaert ER, Ackler SL, Catron ND, Chen J, et al. ABT-199, a potent and selective BCL-2 inhibitor, achieves antitumor activity while sparing platelets. Nat Med. 2013;19:202-8.

36. Yadav B, Wennerberg K, Aittokallio T, Tang J. Searching for drug synergy in complex dose-response landscapes using an interaction potency model. Comput Struct Biotechnol J. 2015;13: 504-13.

37. Chauhan D, Velankar M, Brahmandam M, Hideshima T, Podar K, Richardson $\mathrm{P}$, et al. A novel Bcl-2/Bcl-X(L)/Bcl-w inhibitor ABT737 as therapy in multiple myeloma. Oncogene. 2007;26: 2374-80.

38. Touzeau C, Dousset C, Le Gouill S, Sampath D, Leverson JD, Souers AJ, et al. The Bcl-2 specific BH3 mimetic ABT-199: a promising targeted therapy for $\mathrm{t}(11 ; 14)$ multiple myeloma. Leukemia. 2014;28:210-2.

39. Trudel S, Stewart AK, Li Z, Shu Y, Liang SB, Trieu Y, et al. The Bcl-2 family protein inhibitor, ABT-737, has substantial antimyeloma activity and shows synergistic effect with dexamethasone and melphalan. Clin Cancer Res. 2007;13:621-9.

40. Tse C, Shoemaker AR, Adickes J, Anderson MG, Chen J, Jin S, et al. ABT-263: a potent and orally bioavailable Bcl-2 family inhibitor. Cancer Res. 2008;68:3421-8.

41. Kaufman JL, Gasparetto CJ, Mikhael J, Moreau P, Touzeau C, Vij $\mathrm{R}$, et al. Phase 1 study of venetoclax in combination with dexamethasone as targeted therapy for $\mathrm{t}(11 ; 14)$ relapsed/refractory multiple myeloma. Blood. 2017;130:3131.

42. Kervoelen C, Menoret E, Gomez-Bougie P, Bataille R, Godon C, Marionneau-Lambot $\mathrm{S}$, et al. Dexamethasone-induced cell death is restricted to specific molecular subgroups of multiple myeloma. Oncotarget. 2015;6:26922-34.

43. Matulis SM, Gupta VA, Nooka AK, Hollen HV, Kaufman JL, Lonial S, et al. Dexamethasone treatment promotes Bcl-2 dependence in multiple myeloma resulting in sensitivity to venetoclax. Leukemia. 2016;30:1086-93.

44. Punnoose EA, Leverson JD, Peale F, Boghaert ER, Belmont LD, Tan N, et al. Expression profile of BCL-2, BCL-XL, and MCL-1 predicts pharmacological response to the BCL-2 selective antagonist venetoclax in multiple myeloma models. Mol Cancer Ther. 2016;15:1132-44. 
45. Agarwal R, Chan YC, Tam CS, Hunter T, Vassiliadis D, Teh CE, et al. Dynamic molecular monitoring reveals that SWI-SNF mutations mediate resistance to ibrutinib plus venetoclax in mantle cell lymphoma. Nat Med. 2019;25:119-29.

46. Blombery P, Anderson MA, Gong JN, Thijssen R, Birkinshaw RW, Thompson ER, et al. Acquisition of the recurrent Gly101Val mutation in BCL2 confers resistance to venetoclax in patients with progressive chronic lymphocytic leukemia. Cancer Discov. 2019;9:342-53.

47. van Delft MF, Wei AH, Mason KD, Vandenberg CJ, Chen L, Czabotar PE, et al. The BH3 mimetic ABT-737 targets selective $\mathrm{Bcl}-2$ proteins and efficiently induces apoptosis via Bak/Bax if Mcl-1 is neutralized. Cancer Cell. 2006;10:389-99.

48. Wuilleme-Toumi S, Robillard N, Gomez P, Moreau P, Le Gouill $\mathrm{S}$, Avet-Loiseau $\mathrm{H}$, et al. Mcl-1 is overexpressed in multiple myeloma and associated with relapse and shorter survival. Leukemia. 2005;19:1248-52.

49. Beck IM, Vanden Berghe W, Vermeulen L, Yamamoto KR, Haegeman G, De Bosscher K. Crosstalk in inflammation: the interplay of glucocorticoid receptor-based mechanisms and kinases and phosphatases. Endocr Rev. 2009;30:830-82.
50. Shabestari RM, Safa M, Alikarami F, Banan M, Kazemi A. CREB knockdown inhibits growth and induces apoptosis in human pre-B acute lymphoblastic leukemia cells through inhibition of prosurvival signals. Biomed Pharmacother. 2017;87: 274-9.

51. Carter BZ, Mak PY, Mu H, Zhou H, Mak DH, Schober W, et al. Combined targeting of BCL-2 and BCR-ABL tyrosine kinase eradicates chronic myeloid leukemia stem cells. Sci Transl Med. 2016;8:355ra117.

52. Brioli A, Melchor L, Cavo M, Morgan GJ. The impact of intraclonal heterogeneity on the treatment of multiple myeloma. Br J Haematol. 2014;165:441-54.

53. Caenepeel S, Brown SP, Belmontes B, Moody G, Keegan KS, Chui D, et al. AMG 176, a selective MCL1 inhibitor, is effective in hematologic cancer models alone and in combination with established therapies. Cancer Discov. 2018;8: 1582-97.

54. Ramsey HE, Fischer MA, Lee T, Gorska AE, Arrate MP, Fuller L, et al. A novel MCL1 inhibitor combined with venetoclax rescues venetoclax-resistant acute myelogenous leukemia. Cancer Discov. 2018;8:1566-81. 